\title{
FOUR MAPPINGS WITH A COMMON FIXED POINT ON E-b-METRIC SPACES
}

\author{
Mamta Kamra ${ }^{\# 1}$, Kumari Sarita ${ }^{\# 2}$, Renu Chugh ${ }^{* 3}$ \\ \# Department of Mathematics, Indira Gandhi University, Meerpur (Rewari ). Haryana - 122502, India \\ 1mkhaneja15@gmail.com \\ 2 banteecool@gmail.com \\ *Department of Mathematics, Mahrishi Dayanand University, Rohtak, Haryana - 124001, India. \\ ${ }^{3}$ chughr.1@gmail.com
}

\begin{abstract}
In this paper, we consider E-b-metric space, which is a generalized vector metric space.The metric is Riesz space valued. Here, we prove some results concerning common fixed point for four mappings on E-b-metric spaces. This generalizes the results of Rahimi, Abbas and Rad [4 ].
\end{abstract}

Keyword - E-b-metric space, Riesz space, weak annihilators, weakly compatible, weakly increasing

\section{INTRODUCTION}

Rahimi, Abbas and Rad [15] established few common fixed point results on ordered vector metric spaces for four mappings. Motivated by their work, we extend some of their results of common fixed point theorems for operators defined on E-b-metric space. Altun and Cevik( [7],[8]) have proved some results for vector metric space, which is Riesz space valued metric space. E-b-metric space was defined by Petre [13]. He combined the notions of vector metric space and b-metric space. We recall the basic concepts and notations introduced by Altun and Cevik ([4],[6]) and Petre [13].

We follow notions and terminology by Aliprantis and Border [3] and Luxemburg and Zannen [11] for Riesz spaces.

Definition 1.1. A partial order is a binary relation $\leq$ on a set $\mathrm{X}$ which is reflexive, antisymmetric and transitive .A set with partial order is called a partially ordered set. A partially ordered set $(\mathrm{X}, \leq)$ is said to be linearly ordered or totally ordered or a chain if for each pair $\mathrm{x}, \mathrm{y} \in \mathrm{X}$, we have either $\mathrm{x} \leq \mathrm{y}$ or $\mathrm{y} \leq \mathrm{x}$.

A partially ordered set in which every two elements has a supremum or an infimum is called a lattice.A lattice in $\mathrm{X}$ is said to be complete if every subset has supremum or an infimum and Dedikind complete if every nonempty subset which is bounded above ( below), has a supremum (infimum).

A sequence $\left(b_{n}\right)$ is said to be order Cauchy (o-Cauchy) if there exists a sequence $\left(a_{n}\right)$ in E such that $a_{n} \downarrow 0$ and $\left|\mathrm{b}_{\mathrm{n}}-\mathrm{b}_{\mathrm{n}+\mathrm{p}}\right| \leq \mathrm{a}_{\mathrm{n}}$ holds for $\forall \mathrm{n}$ and $\mathrm{p}$.

Definition 1.2. A real vector space equipped with partial order vector space $(\mathrm{E}, \leq)$ is said to be partially ordered vector space if for $\forall \mathrm{x}, \mathrm{y}, \mathrm{z} \in \mathrm{E}$ and $\alpha>0$,

(i) $\quad \mathrm{x} \leq \mathrm{y}$ implies $\mathrm{x}+\mathrm{z} \leq \mathrm{y}+\mathrm{z}$.

(ii) $\quad \mathrm{x} \leq \mathrm{y}$ impies $\alpha \mathrm{x} \leq \alpha \mathrm{y}$.

Definition1,3. A partially ordered vector space which is also a lattice under its ordering, is called a Riesz space.

Notation: If $\left(\mathrm{x}_{\mathrm{n}}\right)$ is a decreasing sequence in a Riesz space $\mathrm{E}$ such that g.l.b. $\mathrm{x}_{\mathrm{n}}=\mathrm{x}$, we write

$$
\mathrm{X}_{\mathrm{n}} \downarrow \mathrm{X} \text {. }
$$

Definition 1.4. A Riesz space is said to be Archimedean if $\frac{1}{\mathrm{n}} \mathrm{a} \downarrow 0 \quad \forall \mathrm{a} \in \mathrm{E}_{+}$where

$$
\mathrm{E}_{+}=\{\mathrm{a} \in \mathrm{E}: \mathrm{a} \geq 0\} .
$$

Definition 1.5. A sequence $\left(b_{n}\right)$ in a Riesz space $E$ is said to be order convergent to $b$, written as $b_{n} \stackrel{0}{\longrightarrow}$ b or o-lim $b_{n}=b$ if there exists a sequence $\left(a_{n}\right)$ in E satisfying $\mathrm{a}_{\mathrm{n}} \downarrow 0$ and $\left|\mathrm{b}_{\mathrm{n}}-\mathrm{b}\right| \leq \mathrm{a}_{\mathrm{n}} \forall \mathrm{n}$ where $|\mathrm{b}|=\mathrm{b} \mathrm{v}-\mathrm{b}$.

A Riesz space $\mathrm{E}$ is said to be $\mathrm{o}-$ complete if every $\mathrm{o}-$ Cauchy sequence is $\mathrm{o}$-convergent.

Lemma 1.6.[6] If $\mathrm{E}$ is a Riesz space and $\mathrm{a} \leq \mathrm{ka}$ holds where $\mathrm{a} \in \mathrm{E}_{+}, \mathrm{k} \in[0,1)$ then $\mathrm{a}=0$.

Example 1.7.. $\mathrm{R}^{2}$ with coordinatewise ordering is an Archimedean Riesz space.

Definition 1.8 . Let $X$ be a nonempty set and $E$ be a Riesz space. . Then function

$\mathrm{d}: \mathrm{X} \times \mathrm{X} \rightarrow \mathrm{E}$ is said to be a vector metric if it satisfies : 
(a) $\quad d(x, y)=$ o iff $x=y$

(b) $\quad d(x, y) \leq d(x, w)+d(y, w) \forall x, y, w \in X$.

Then $(X, d, E)$ is said to be a vector metric space or E-metric space. Vector metric Space generalizes metric space.

For $\mathrm{x}, \mathrm{y}, \mathrm{z}, \mathrm{w}$ of a vector metric space, the following statements hold:

(i) $0 \leq d(x, y)$

(ii) $d(x, y)=d(y, x)$

(iii) $|d(x, w)-d(y, w)| \leq d(x, y)$

(iv) $|d(x, w)-d(y, z)| \leq d(x, y)+d(w, z)$

A sequence $\left(x_{n}\right)$ in a vector metric space $(X, d, E)$ is said to be vectorial convergent or

E-convergent to some $\mathrm{x} \in \mathrm{E}$, written as $\mathrm{x}_{\mathrm{n}} \stackrel{\text { d.E }}{\longrightarrow} \mathrm{x}$ if $\exists\left(\mathrm{a}_{\mathrm{n}}\right)$ in $\mathrm{E}$ such that $\mathrm{a}_{\mathrm{n}} \downarrow 0$ and $\mathrm{d}\left(\mathrm{x}_{\mathrm{n}}, \mathrm{x}\right) \leq \mathrm{a}_{\mathrm{n}} \forall \mathrm{n}$.

A sequence $\left(x_{n}\right)$ is said to be E-Cauchy sequence if there exists a sequence $\left(a_{n}\right)$ in $E$ such that $\mathrm{a}_{\mathrm{n}} \downarrow 0$ and $\mathrm{d}\left(\mathrm{x}_{\mathrm{n}}, \mathrm{x}_{\mathrm{n}+\mathrm{p}}\right) \leq \mathrm{a}_{\mathrm{n}}$ holds $\forall \mathrm{n}$ and $\mathrm{p}$.

$A$ vector metric space $\mathrm{X}$ is called $\mathrm{E}$-complete if each $\mathrm{E}$-Cauchy sequence in $\mathrm{X}$, vectorial converges to a limit in $\mathrm{X}$.

When $\mathrm{E}=\mathrm{R}$ (the set of real numbers) the concepts of vectorial convergence and metric convergence, E-Cauchy sequence and Cauchy sequence are the same.

If $\mathrm{X}=\mathrm{E}$ and $\mathrm{d}$ is the absolute valued vector metric on $\mathrm{X}$, then the concepts of vectorial convergence and order convergence are the same.

Example 1.9. A Riesz space $E$ is a vector metric space with $d: E \times E \rightarrow E$ defined by $\mathrm{d}(\mathrm{x}, \mathrm{y})=|\mathrm{x}-\mathrm{y}| \forall \mathrm{x}, \mathrm{y} \in \mathrm{X}$, is called absolute valued metric on $\mathrm{E}$.

Definition 1.10. Let $\mathrm{X}$ be a non-empty set and let $\mathrm{s} \geq 1$ be a given real number. $\mathrm{A}$ function

$d: X \times X \rightarrow R_{+}$is called $b$ - metric if it satisfies the following conditions :

(i) $d(x, y)=0$ iff $x=y$

(ii) $\quad d(x, y)=d(y, x)$

(iii) $d(x, w) \leq s[d(x, y)+d(y, w)]$ for all $x, y, w \in X$.

Fixed point theorems on $b$-metric spaces have been studied by several authors, one can see ([10],[12]).

I.R. Petre [13] defined vector b-metric or E-b-metric $\$$ pace as follows :

Definition 1.11. Let $\mathrm{X}$ be a non-empty set and $\mathrm{s} \geq 1$. A function $\mathrm{d}: \mathrm{X} \times \mathrm{X} \rightarrow \mathrm{E}_{+}$is called $\mathrm{E}-\mathrm{b}-$ metric if for any $\mathrm{x}, \mathrm{y}, \mathrm{w} \in \mathrm{X}$, the following conditions hold:

(a) $d(x, y)=0$ iff $x=y$

(b) $\mathrm{d}(\mathrm{x}, \mathrm{y})=\mathrm{d}(\mathrm{y}, \mathrm{x})$

(c) $d(x, w) \leq s[d(x, y)+d(y, w)]$

Then $(X, d, E)$ is said to be $E-b-$ metric space.

Below we give some examples of $\mathrm{E}-\mathrm{b}$-metric spaces.

Example 1.12. Let $X=[0,1], d:[0,1] \times[0,1] \rightarrow R^{2}$ defined by $d(x, y)=\left(\alpha|x-y|^{2}, \beta|x-y|^{2}\right)$ where $\alpha, \beta \geq 0$ and $\alpha+\beta>0$, then $\left(\mathrm{X}, \mathrm{d}, \mathrm{R}^{2}\right)$ is E-b-metric space.

Example 1.13. Let $\mathrm{X}=\mathrm{L}^{\mathrm{P}}[0,1]$ with $0<\mathrm{p}<1$ and $\mathrm{E}=\mathrm{R}^{2}$.

Let $\mathrm{d}: \mathrm{L}^{\mathrm{P}}[0,1] \times \mathrm{L}^{\mathrm{P}}[0,1] \rightarrow \mathrm{R}^{2}+$ be defined by

$$
\mathrm{d}(\mathrm{f}, \mathrm{g})=\left(\alpha\|\mathrm{f}-\mathrm{g}\|_{\mathrm{p}}, \beta\|\mathrm{f}-\mathrm{g}\|_{\mathrm{p}}\right)
$$

where $\alpha, \beta \geq 0$ and $\alpha+\beta>0$.

Then it is easy to see that

$$
d(f, h) \leq 2[d(f, g)+d(g, h)] .
$$


Hence $\left(\mathrm{X}, \mathrm{d}, \mathrm{R}^{2}\right)$ is E-b-metric space with parameter $\mathrm{s}=2^{\frac{1}{p}}>1$.

Likewise, if $X=1_{p}, 0<p<1$, and $d: 1_{p} \times 1_{p} \rightarrow R^{2}+$ defined as

$$
\mathrm{d}(\mathrm{x}, \mathrm{y})=\left(\alpha\|\mathrm{x}-\mathrm{y}\|_{\mathrm{p}}, \beta\|\mathrm{x}-\mathrm{y}\|_{\mathrm{p}}\right),
$$

where $\alpha, \beta \geq 0$ and $\alpha+\beta>0$, then ( $\left.\mathrm{X}, \mathrm{d}, \mathrm{R}^{2}\right)$ is E-b-metric space

with parameter

$$
\mathrm{s}=2^{\frac{1}{p}}>1 .
$$

Example 1.14. Let $\mathrm{X}=\mathrm{C}[0,1]=\mathrm{E}$. Define $\mathrm{d}: \mathrm{X} \times \mathrm{X} \rightarrow \mathrm{E}_{+}$as

$$
\mathrm{d}(\mathrm{f}, \mathrm{g})=(\mathrm{f}-\mathrm{g})^{\mathrm{p}}, \mathrm{p}>1 \text {. }
$$

Then $(\mathrm{X}, \mathrm{d}, \mathrm{E})$ is $\mathrm{E}-\mathrm{b}$-metric space with parameter $\mathrm{s}=2>1$. Since the function

$t^{p}(p>1)$ is convex, we have

$$
(x+y)^{p} \leq 2^{p-1}\left(x^{p}+y^{p}\right)
$$

Therefore

$$
\begin{aligned}
d(f, h)=(f-h)^{p}= & (f-g+g-h)^{p} \leq 2^{p-1}\left[(f-g)^{p}+(g-h)^{p}\right] \\
& =2^{p-1}[d(f, g)+d(g, h)] .
\end{aligned}
$$

Thus relaxed triangular inequality holds with $\mathrm{s}=2^{\mathrm{p}-1}>1$.

We give below an example of E-b-metric space which is not a metric space.

Example 1.15. Let $\mathrm{X}=\{0,1,2\}, \mathrm{E}=\mathrm{R}^{2}$ and $\mathrm{d}: \mathrm{X} \times \mathrm{X} \rightarrow \mathrm{R}^{2}$ be defined as

$$
\begin{aligned}
& \mathrm{d}(0,1)=\mathrm{d}(1,0)=(1,1) \\
& \mathrm{d}(1,2)=\mathrm{d}(2,1)=(1,1) \\
& \mathrm{d}(0,2)=\mathrm{d}(2,0)=(4,4)
\end{aligned}
$$

Since the inequality $d(0,2) \leq d(0,1)+d(1,2)$ is not satisfied, $(X, d, E)$ is not a metric space. However, it is E-b-metric space for $\mathrm{s}=2$.

Let (X, d, E) be E-b metric space and $P$ and $Q$ be self maps on $X$.

Definition 1.16[15]. Let $\mathrm{y}=\mathrm{Px}=\mathrm{Qx}$ for some $\mathrm{x} \in \mathrm{X}$ then $\mathrm{y}$ is said to be a point of coincidence and $\mathrm{x} a$ coincidence point of $\mathrm{P}$ and $\mathrm{Q}$.

Definition 1.17[15]. The maps $\mathrm{P}$ and $\mathrm{Q}$ are called weakly compatible if they commute at every coincidence point.

Lemma 1.18[15]. The maps $\mathrm{P}$ and $\mathrm{Q}$ are weakly compitable maps $\mathrm{X}$ If $\mathrm{P}$ and $\mathrm{Q}$ have a unique point of coincidence $\mathrm{c}=\mathrm{Pc}=\mathrm{Qc}$, then $\mathrm{c}$ is the unique common fixed point of $\mathrm{Pand} \mathrm{Q}$.

Let $(\mathrm{X}, \preccurlyeq)$ be a partially ordered set and $\mathrm{P}$ and $\mathrm{Q}$ are self maps on $\mathrm{X}$.

Definition 1.19[15]. The pair (P, Q) of maps is said to be weakly increasing if $\mathrm{Px} \preccurlyeq \mathrm{QPx}$ and Qx $\preccurlyeq \mathrm{PQx}$ $\forall \mathrm{x} \in \mathrm{X}$.

Definition 1.20[15]. The pair (P, Q) is said to be partially weakly increasing if $\mathrm{Px} \preccurlyeq \mathrm{QPx}$

$\forall \mathrm{x} \in \mathrm{X}$.

Observe that the pair $(\mathrm{P}, \mathrm{Q})$ is weakly increasing iff ordered pair $(\mathrm{P}, \mathrm{Q})$ and $(\mathrm{Q}, \mathrm{P})$ are partially weakly increasing.

Definition 1.21[15]. The mapping $\mathrm{P}$ is called a weak annihilator of $\mathrm{Q}$ if $\mathrm{PQx} \preccurlyeq \mathrm{x} \forall \mathrm{x} \in \mathrm{X}$.

Definition 1.22[15]. The mapping $P$ is called dominating if $x \leqslant P x \forall x \in X$.

\section{MAIN RESULTS}

In this section, we prove some common fixed point theorems for four mappings in $\mathrm{E}-\mathrm{b}-$-metric space.

Theorem 2.1. Let $\mathrm{X}$ be an $\mathrm{E}-\mathrm{b}-$-metric space with $\mathrm{E}$ is Archimedean. Suppose the mapping A, B, G and $\mathrm{H}$ be self-maps on $X,(H, A)$ and $(G, B)$ be partially weakly increasing with $A(X) \subseteq H(X)$ and $B(X) \subseteq G(X)$. Further the dominating maps A and B are weak annihilators of $\mathrm{H}$ and $\mathrm{G}$, respectively. Suppose the mappings A, B, G, $\mathrm{H}: \mathrm{X} \rightarrow \mathrm{X}$ satisfy the following condition :

$$
\mathrm{d}(\mathrm{Ax}, \mathrm{By}) \leq \mathrm{bN}_{\mathrm{x}, \mathrm{y}}(\mathrm{A}, \mathrm{B}, \mathrm{G}, \mathrm{H}) \quad \forall \mathrm{x}, \mathrm{y} \in \mathrm{X},
$$




$$
\text { where } \mathrm{b}<\frac{1}{s(s+1)} \text { and }
$$

$\mathrm{N}_{\mathrm{x}, \mathrm{y}}(\mathrm{A}, \mathrm{B}, \mathrm{G}, \mathrm{H}) \in\{\mathrm{d}(\mathrm{Gx}, \mathrm{Hy}), \mathrm{d}(\mathrm{Ax}, \mathrm{Gx}), \mathrm{d}(\mathrm{By}, \mathrm{Hy}), \mathrm{d}(\mathrm{Ax}, \mathrm{Hy}), \mathrm{d}(\mathrm{By}, \mathrm{Gx})\}$

Let $\left\{\mathrm{x}_{\mathrm{n}}\right\}$ be a nondecreasing sequence with $\mathrm{x}_{\mathrm{n}} \leq \mathrm{y}_{\mathrm{n}} \forall \mathrm{n}$ and $\mathrm{y}_{\mathrm{n}} \stackrel{\text { d.E }}{\longrightarrow} m$ gives that $\mathrm{x}_{\mathrm{n}} \leq \mathrm{m}$, the pairs $\{\mathrm{A}, \mathrm{G}\}$ and $\{\mathrm{B}, \mathrm{H}\}$ are given to be weakly compatible and if one of $\mathrm{A}(\mathrm{X}), \mathrm{B}(X)$, $G(X)$ or $H(X)$ is E-complete subspace of $X$ then $A, B, G$ and $H$ have a unique common fixed point.

Proof : Let $\mathrm{x}_{0}$ be arbitrary point of $X$. Since $A(X) \subseteq H(X)$ and $B(X) \subseteq G(X)$, we can choose $x_{1} \in X$ such that $\mathrm{Ax}_{0}=\mathrm{Hx}_{1}, \mathrm{x}_{2} \in \mathrm{X}$ such that $\mathrm{Bx}_{1}=\mathrm{Gx}_{2}$. Continuing this process, construct a sequence $\left\{\mathrm{y}_{\mathrm{n}}\right\}$, which is defined as :

$$
\mathrm{y}_{2 \mathrm{n}-1}=\mathrm{Hx}_{2 \mathrm{n}-1}=\mathrm{Ax}_{2 \mathrm{n}-2} \text { and } \mathrm{y}_{2 \mathrm{n}}=\mathrm{Gx}_{2 \mathrm{n}}=\mathrm{Bx}_{2 \mathrm{n}-1}, \quad \mathrm{n} \geq 0 .
$$

By given assumptions,

$$
\mathrm{x}_{2 \mathrm{n}-2} \leq \mathrm{Ax}_{2 \mathrm{n}-2}=\mathrm{Hx}_{2 \mathrm{n}-1} \leq \mathrm{AHx}_{2 \mathrm{n}-1} \leq \mathrm{x}_{2 \mathrm{n}-1} \text { and } \mathrm{x}_{2 \mathrm{n}-1} \leq \mathrm{Bx}_{2 \mathrm{n}-1}=\mathrm{Sx}_{2 \mathrm{n}} \leq \mathrm{BGx}_{2 \mathrm{n}} \leq \mathrm{x}_{2 \mathrm{n}} .
$$

Thus, for all $\mathrm{n}$ we have, $\mathrm{x}_{\mathrm{n}} \leq \mathrm{x}_{\mathrm{n}+1}$.

Firstly, we prove that

$$
\mathrm{d}\left(\mathrm{y}_{2 \mathrm{n}+1}, \mathrm{y}_{2 \mathrm{n}+2}\right) \leq \lambda \mathrm{d}\left(\mathrm{y}_{2 \mathrm{n}}, \mathrm{y}_{2 \mathrm{n}+1}\right) \quad \mathrm{n}, \text { where } \lambda<1
$$

From (1), we have

$$
\mathrm{d}\left(\mathrm{y}_{2 \mathrm{n}+1}, \mathrm{y}_{2 \mathrm{n}+2}\right)=\mathrm{d}\left(\mathrm{Ax}_{2 \mathrm{n}}, \mathrm{Bx}_{2 \mathrm{n}+1}\right) \leq \mathrm{bN}_{\mathrm{x}_{2 \mathrm{n}}, \mathrm{x}_{2 \mathrm{n}+1}}(\mathrm{~A}, \mathrm{~B}, \mathrm{G}, \mathrm{H}) \text { for } \mathrm{n}=0,1,2,3 \ldots .
$$

Since $\mathrm{N}_{\mathrm{x}_{2 \mathrm{n}}, \mathrm{x}_{2 \mathrm{n}+1}}(\mathrm{~A}, \mathrm{~B}, \mathrm{G}, \mathrm{H}) \in\left\{\mathrm{d}\left(\mathrm{Gx}_{2 \mathrm{n}}, \mathrm{Hx}_{2 \mathrm{n}+1}\right), \mathrm{d}\left(\mathrm{Ax}_{2 \mathrm{n}}, \mathrm{Gx}_{2 \mathrm{n}}\right), \mathrm{d}\left(\mathrm{Bx}_{2 \mathrm{n}+1}, \mathrm{Hx}_{2 \mathrm{n}+1}\right)\right.$,

$$
\left.\mathrm{d}\left(\mathrm{Ax}_{2 \mathrm{n}}, \mathrm{Hx}_{2 \mathrm{n}+1}\right), \mathrm{d}\left(\mathrm{Bx}_{2 \mathrm{n}+1}, \mathrm{Gx}_{2 \mathrm{n}}\right)\right\}
$$

$=\left\{d\left(y_{2 n}, y_{2 n+1}\right), d\left(y_{2 n+1}, y_{2 n}\right), d\left(y_{2 n+2}, y_{2 n+1}\right), d\left(y_{2 n+1}, y_{2 n+1}\right), d\left(y_{2 n+2}, y_{2 n}\right)\right\}$

$$
=\left\{d\left(y_{2 n}, y_{2 n+1}\right), d\left(y_{2 n+1}, y_{2 n+2}\right), d\left(y_{2 n}, y_{2 n+2}\right)\right\}
$$

If $\mathrm{N}_{\mathrm{x}_{2 \mathrm{n}}, \mathrm{x}_{2 \mathrm{n}+1}}(\mathrm{~A}, \mathrm{~B}, \mathrm{G}, \mathrm{H})=\mathrm{d}\left(\mathrm{y}_{2 \mathrm{n}}, \mathrm{y}_{2 \mathrm{n}+1}\right)$, then clearly ( 3) holds.

If $\mathrm{N}_{\mathrm{x}_{2 n}, \mathrm{x}_{2 n+1}}(\mathrm{~A}, \mathrm{~B}, \mathrm{G}, \mathrm{H})=\mathrm{d}\left(\mathrm{y}_{2 \mathrm{n}+1}, \mathrm{y}_{2 \mathrm{n}+2}\right)$, thus by Lemma 1.6

$$
\mathrm{d}\left(\mathrm{y}_{2 \mathrm{n}+1}, \mathrm{y}_{2 \mathrm{n}+2}\right)=0 \text {, and so (3) holds. }
$$

Now assume that $\mathrm{N}_{\mathrm{x}_{2 \mathrm{n}}, \mathrm{x}_{2 \mathrm{n}+1}}(\mathrm{~A}, \mathrm{~B}, \mathrm{G}, \mathrm{H})=\mathrm{d}\left(\mathrm{y}_{2 \mathrm{n}}, \mathrm{y}_{2 \mathrm{n}+2}\right)$,

Thus,

$$
\begin{aligned}
& d\left(y_{2 n+1}, y_{2 n+2}\right) \leq b d\left(y_{2 n}, y_{2 n+2}\right) \leq b s\left[d\left(y_{2 n}, y_{2 n+1}\right)+d\left(y_{2 n+1}, y_{2 n+2}\right)\right] \\
&(1-b s) d\left(y_{2 n+1}, y_{2 n+2}\right) \leq b s d\left(y_{2 n}, y_{2 n+1}\right) \\
& \leq\left(\frac{b s}{1-b s}\right)\left[d\left(y_{2 n}, y_{2 n+1}\right)\right] \\
&=\lambda d\left(y_{2 n}, y_{2 n+1}\right), \text { where } \lambda=\left(\frac{b s}{1-b s}\right)
\end{aligned}
$$

Thus $\mathrm{d}\left(\mathrm{y}_{\mathrm{n}}, \mathrm{y}_{\mathrm{n}+1}\right) \leq \lambda^{\mathrm{n}} \mathrm{d}\left(\mathrm{y}_{0}, \mathrm{y}_{1}\right)$ where $\lambda \in\left\{b, \frac{b s}{1-b s}\right\}$

Therefore $\forall \mathrm{n}$ and $\mathrm{p}$,

$$
\begin{aligned}
\mathrm{d}\left(\mathrm{y}_{\mathrm{n}}, \mathrm{y}_{\mathrm{n}+\mathrm{p}}\right) & \leq \mathrm{sd}\left(\mathrm{y}_{\mathrm{n}}, \mathrm{y}_{\mathrm{n}+1}\right)+\mathrm{s}^{2} \mathrm{~d}\left(\mathrm{y}_{\mathrm{n}+1}, \mathrm{y}_{\mathrm{n}+2}\right)+\mathrm{s}^{3} \mathrm{~d}\left(\mathrm{y}_{\mathrm{n}+2}, \mathrm{y}_{\mathrm{n}+3}\right)+\ldots \ldots+\mathrm{s}^{\mathrm{p}} \mathrm{d}\left(\mathrm{y}_{\mathrm{n}+\mathrm{p}-1}, \mathrm{y}_{\mathrm{n}+\mathrm{p}}\right) \\
& \leq \mathrm{s} \lambda^{\mathrm{n}} \mathrm{d}\left(\mathrm{y}_{0}, \mathrm{y}_{1}\right)+\mathrm{s}^{2} \lambda^{\mathrm{n}+1} \mathrm{~d}\left(\mathrm{y}_{0}, \mathrm{y}_{1}\right)+\ldots \ldots \ldots \ldots+\mathrm{s}^{\mathrm{p}} \lambda^{\mathrm{n}+\mathrm{p}-1} \mathrm{~d}\left(\mathrm{y}_{0}, \mathrm{y}_{1}\right) \\
= & s \lambda^{n}\left(\frac{1-(\mathrm{s} \lambda)^{p}}{1-s \lambda}\right) \mathrm{d}\left(\mathrm{y}_{0}, \mathrm{y}_{1}\right) \leq\left(\frac{s \lambda^{n}}{1-s \lambda}\right) \mathrm{d}\left(\mathrm{y}_{0}, \mathrm{y}_{1}\right), \text { note that } \mathrm{s} \lambda<1
\end{aligned}
$$

It is given that $\mathrm{E}$ is Archimedean, so the sequence $\left(\mathrm{y}_{\mathrm{n}}\right)$ is an $\mathrm{E}-$ Cauchy . It is assumed that $\mathrm{G}(\mathrm{X})$ is $\mathrm{E}$-complete, $\exists \mathrm{u} \in \mathrm{G}(\mathrm{X})$ such that 


$$
\mathrm{Gx}_{2 \mathrm{n}}=\mathrm{y}_{2 \mathrm{n}} \stackrel{\text { d.E. }}{\longrightarrow} \mathrm{u}
$$

Thus, there exists a sequence $\left\{\mathrm{e}_{\mathrm{n}}\right\}$ in E such that $\mathrm{e}_{\mathrm{n}} \downarrow 0$ and $\mathrm{d}\left(\mathrm{Gx}_{2 \mathrm{n}}, \mathrm{u}\right) \leq \mathrm{e}_{\mathrm{n}}$. Further we can find out $a v \in X$ such that $G v=u$. Now it remains to prove that $A v=u$. Although $\mathrm{x}_{2 \mathrm{n}+1} \leq \mathrm{Bx}_{2 \mathrm{n}+1}$ and $\mathrm{Bx}_{2 \mathrm{n}+1} \stackrel{\text { d.E. }}{\longrightarrow} \mathrm{u}$ which implies that $\mathrm{x}_{2 \mathrm{n}+1} \leq \mathrm{u}$ and $\mathrm{u} \leq \mathrm{Bu}=\mathrm{BGv} \leq \mathrm{v}$ which implies that $\mathrm{x}_{2 \mathrm{n}+1} \leq \mathrm{v}$

Consider

$$
\mathrm{d}(\mathrm{Av}, \mathrm{u}) \leq \mathrm{sd}\left(\mathrm{Av}, \mathrm{Bx}_{2 \mathrm{n}+1}\right)+\mathrm{sd}\left(\mathrm{Bx}_{2 \mathrm{n}+1}, \mathrm{u}\right) \leq \mathrm{sbN}_{\mathrm{v}, \mathrm{x}_{2 n+1}}(\mathrm{~A}, \mathrm{~B}, \mathrm{G}, \mathrm{H})+\mathrm{se}_{\mathrm{n}+1}
$$

where $b_{v} \mathrm{x}_{2 \mathrm{n}+1}(\mathrm{~A}, \mathrm{~B}, \mathrm{G}, \mathrm{H}) \in\left\{\mathrm{d}\left(\mathrm{Gv}, \mathrm{Hx}_{2 \mathrm{n}+1}\right), \mathrm{d}(\mathrm{Av}, \mathrm{Gv}), \mathrm{d}\left(\mathrm{Bx}_{2 \mathrm{n}+1}, \mathrm{Hx}_{2 \mathrm{n}+1}\right), \mathrm{d}\left(\mathrm{Av}, \mathrm{Hx}_{2 \mathrm{n}+1}\right), \mathrm{d}\left(\mathrm{Bx}_{2 \mathrm{n}+1}, \mathrm{Gv}\right)\right\}$ n.

There are five possibilities:

Case $1: \mathrm{d}(\mathrm{Av}, \mathrm{u}) \leq \operatorname{sbd}\left(\mathrm{Gv}, \mathrm{Hx}_{2 \mathrm{n}+1}\right)+\mathrm{se}_{\mathrm{n}+1} \leq \mathrm{sbe}_{\mathrm{n}+1}+\mathrm{se}_{\mathrm{n}+1} \leq \mathrm{s}(\mathrm{b}+1) \mathrm{e}_{\mathrm{n}}$.

Case $2: \mathrm{d}(\mathrm{Av}, \mathrm{u}) \leq \operatorname{sbd}(\mathrm{Av}, \mathrm{Gv})+\mathrm{se}_{\mathrm{n}+1}$

$$
\leq \operatorname{sbd}(\mathrm{Av}, \mathrm{u})+\mathrm{se}_{\mathrm{n}+1}
$$

$(1-\mathrm{sb}) \mathrm{d}(\mathrm{Av}, \mathrm{u}) \leq \mathrm{se}_{\mathrm{n}+1}$

$$
\begin{aligned}
\mathrm{d}(\mathrm{Av}, \mathrm{u}) & \leq \frac{\mathrm{s}}{(1-\mathrm{sb})} \mathrm{e}_{\mathrm{n}+1} \\
& \leq \frac{\mathrm{s}}{(1-\mathrm{sb})} \mathrm{e}_{\mathrm{n}}
\end{aligned}
$$

Case $3: d(A v, u) \leq \operatorname{sbd}\left(B_{2 n+1}, H_{2 n+1}\right)+s_{n+1}$

$$
\begin{aligned}
& \mathrm{d}(\mathrm{Av}, \mathrm{u}) \leq \mathrm{sb}\left[\mathrm{sd}\left(\mathrm{Bx}_{2 \mathrm{n}+1}, \mathrm{u}\right)+\mathrm{sd}\left(\mathrm{Hx}_{2 \mathrm{n}+1}, \mathrm{u}\right)\right]+\mathrm{se}_{\mathrm{n}+1} \\
& \mathrm{~d}(\mathrm{Av}, \mathrm{u}) \leq \mathrm{s}^{2} \mathrm{be}_{\mathrm{n}+1}+\mathrm{s}^{2} \mathrm{be}_{\mathrm{n}+1}+\mathrm{se}_{\mathrm{n}+1} \\
& \leq 2 \mathrm{~s}^{2} \mathrm{be}_{\mathrm{n}+1}+\mathrm{se}_{\mathrm{n}+1} \leq \mathrm{s}(2 \mathrm{bs}+1) \mathrm{e}_{\mathrm{n}}
\end{aligned}
$$

Case 4: d(Av, u) $\leq \operatorname{sbd}\left(\mathrm{Av}, \mathrm{Hx}_{2 \mathrm{n}+1}\right)+\mathrm{se}_{\mathrm{n}+1}$

$$
\leq \mathrm{sb}\left[\mathrm{sd}(\mathrm{Av}, \mathrm{u})+\mathrm{sd}\left(\mathrm{u}, \mathrm{Hx}_{2 \mathrm{n}+1}\right)\right]+\mathrm{se}_{\mathrm{n}+1}
$$

$\left(1-s^{2} b\right) d(A v, u) \leq s^{2} b e_{n+1}+s_{n+1}$

$$
\mathrm{d}(\mathrm{Av}, \mathrm{u}) \leq \frac{\mathrm{s}(\mathrm{sb}+1)}{\left(1-\mathrm{s}^{2} \mathrm{~b}\right)} \mathrm{e}_{\mathrm{n}}
$$

Case 5 : $d(A v, u) \leq \operatorname{sbd}\left(B_{2 n+1}, G v\right)+\operatorname{se}_{n+1}$

$$
\leq \mathrm{sbe}_{\mathrm{n}+1}+\mathrm{se}_{\mathrm{n}+1} \leq \mathrm{s}(\mathrm{b}+1) \mathrm{e}_{\mathrm{n}}
$$

It is given that g.l.b. of sequences on the R.H.S. are zero, then $d(A v, u)=0$, i.e. $A v=u$.

Thus $\mathrm{Av}=\mathrm{Gv}=\mathrm{u}$

However $\mathrm{u} \in \mathrm{A}(\mathrm{X}) \subseteq \mathrm{H}(\mathrm{X})$, so there exists $\mathrm{w} \in \mathrm{X}$ such that $\mathrm{Hw}=\mathrm{u}$

Now, we show that $\mathrm{Bw}=\mathrm{u}$. As $\mathrm{x}_{2 \mathrm{n}} \leq \mathrm{Ax}_{2 \mathrm{n}}$ and $\mathrm{Ax}_{2 \mathrm{n}} \stackrel{\text { d.E. }}{\longrightarrow} \mathrm{u}$ implies that $\mathrm{x}_{2 \mathrm{n}} \leq \mathrm{u}$ and $\mathrm{u} \leq \mathrm{Au}=\mathrm{AHw} \leq \mathrm{w}$ implies that $\mathrm{x}_{2 \mathrm{n}} \leq \mathrm{w}$.

Consider

$$
\mathrm{d}(\mathrm{u}, \mathrm{Bw}) \leq \mathrm{sd}\left(\mathrm{u}, \mathrm{Ax}_{2 \mathrm{n}}\right)+\operatorname{sd}\left(\mathrm{Ax}_{2 \mathrm{n}}, \mathrm{Bw}\right) \leq \mathrm{se}_{\mathrm{n}}+\operatorname{sbN}_{\mathrm{x}_{2 \mathrm{n}, \mathrm{w}}}(\mathrm{A}, \mathrm{B}, \mathrm{G}, \mathrm{H})
$$

where $\mathrm{N}_{\mathrm{x}_{2 \mathrm{n}, \mathrm{w}}}(\mathrm{A}, \mathrm{B}, \mathrm{G}, \mathrm{H}) \in\left\{\mathrm{d}\left(\mathrm{Gx}_{2 \mathrm{n}}, \mathrm{Hw}\right), \mathrm{d}\left(\mathrm{Ax}_{2 \mathrm{n}}, \mathrm{Gx}_{2 \mathrm{n}}\right), \mathrm{d}(\mathrm{Bw}, \mathrm{Hw}), \mathrm{d}\left(\mathrm{Ax}_{2 \mathrm{n}}, \mathrm{Hw}\right)\right.$,

$$
\left.\mathrm{d}\left(\mathrm{Bw}, \mathrm{Gx}_{2 \mathrm{n}}\right)\right\} \quad \mathrm{n} \text {. }
$$

There are five possibilities:

Case $1: \mathrm{d}(\mathrm{u}, \mathrm{Bw}) \leq \mathrm{se}_{\mathrm{n}}+\operatorname{sbd}\left(\mathrm{Gx}_{2 \mathrm{n}}, \mathrm{Hw}\right) \leq \mathrm{se}_{\mathrm{n}}+\operatorname{sbd}\left(\mathrm{Gx}_{2 \mathrm{n}}, \mathrm{u}\right) \leq \mathrm{s}(\mathrm{b}+1) \mathrm{e}_{\mathrm{n}}$.

Case $2: \mathrm{d}(\mathrm{u}, \mathrm{Bw}) \leq \mathrm{se}_{\mathrm{n}}+\operatorname{sbd}\left(\mathrm{Ax}_{2 \mathrm{n}}, \mathrm{Gx}_{2 \mathrm{n}}\right)$,

$$
\begin{aligned}
& \leq \mathrm{se}_{\mathrm{n}}+\mathrm{sb}\left[\mathrm{sdd}\left(\mathrm{Ax}_{2 \mathrm{n}}, \mathrm{u}\right)+\mathrm{sd}\left(\mathrm{u}, \mathrm{Gx}_{2 \mathrm{n}}\right)\right] \\
& \leq \mathrm{se}_{\mathrm{n}}+\mathrm{s}^{2} \mathrm{be}_{\mathrm{n}}+\mathrm{s}^{2} \mathrm{be}_{\mathrm{n}} \\
& \leq \mathrm{s}(1+2 \mathrm{sb}) \mathrm{e}_{\mathrm{n}}
\end{aligned}
$$

Case $3: \mathrm{d}(\mathrm{u}, \mathrm{Bw}) \leq \mathrm{se}_{\mathrm{n}}+\operatorname{sbd}(\mathrm{Bw}, \mathrm{Hw}) \leq \mathrm{se}_{\mathrm{n}}+\operatorname{sbd}(\mathrm{Bw}, \mathrm{u})$

$(1-\mathrm{sb}) \mathrm{d}(\mathrm{u}, \mathrm{Bw}) \leq \mathrm{se}_{\mathrm{n}}$ 


$$
\mathrm{d}(\mathrm{u}, \mathrm{Bw}) \leq \frac{\mathrm{s}}{(1-\mathrm{sb})} \mathrm{e}_{\mathrm{n}}
$$

Case $4: d(u, B w) \leq \operatorname{se}_{n}+\operatorname{sbd}\left(A_{2 n}, H w\right)$

$$
\begin{aligned}
& \leq \mathrm{se}_{\mathrm{n}}+\operatorname{sbd}\left(\mathrm{Ax}_{2 \mathrm{n}}, \mathrm{u}\right) \\
& \leq \mathrm{se}_{\mathrm{n}}+\mathrm{sbe}_{\mathrm{n}}
\end{aligned}
$$

$\mathrm{d}(\mathrm{u}, \mathrm{Bw}) \leq \mathrm{s}(1+\mathrm{b}) \mathrm{e}_{\mathrm{n}}$

Case $5: d(u, B w) \leq \operatorname{se}_{n}+\operatorname{sbd}\left(B w, G_{2 n}\right)$

$$
\leq \mathrm{se}_{\mathrm{n}}+\mathrm{sb}\left[\mathrm{sd}(\mathrm{Bw}, \mathrm{u})+\mathrm{sd}\left(\mathrm{u}, \mathrm{Gx}_{2 \mathrm{n}}\right)\right]
$$

$\left(1-s^{2} b\right) d(u, B w) \leq \mathrm{se}_{n}+s^{2} b e_{n}$

$$
\mathrm{d}(\mathrm{u}, \mathrm{Bw}) \leq \frac{\mathrm{s}(1+\mathrm{sb})}{\left(1-\mathrm{s}^{2} \mathrm{~b}\right)} \mathrm{e}_{\mathrm{n}}
$$

It is given that g.l.b. of sequences on the L.H.S. are zero, then $\mathrm{d}(\mathrm{u}, \mathrm{Bw})=0$, that is $\mathrm{Bw}=\mathrm{u}$. $\quad$ Therefore $\mathrm{Bw}=$ $\mathrm{Hw}=\mathrm{u}$. Consequently $\{\mathrm{A}, \mathrm{G}\}$ and $\{\mathrm{B}, \mathrm{H}\}$ have a common point of coincidence belonging to $\mathrm{X}$.

Now, if $\{A, G\}$ and $\{B, H\}$ are given to be weakly compatible,

$$
\begin{gathered}
\mathrm{Au}=\mathrm{AGv}=\mathrm{GAv}=\mathrm{Gu} \text { and } \\
\mathrm{Bu}=\mathrm{BHw}=\mathrm{HBw}=\mathrm{Hu}=\mathrm{m}_{2} \text { (say) }
\end{gathered}
$$

Now,

$$
\mathrm{d}\left(\mathrm{m}_{1}, \mathrm{~m}_{2}\right)=\mathrm{d}(\mathrm{Au}, \mathrm{Bu}) \leq \mathrm{bN}_{\mathrm{u}, \mathrm{u}}(\mathrm{A}, \mathrm{B}, \mathrm{G}, \mathrm{H})
$$

where $\mathrm{N}_{\mathrm{u}, \mathrm{u}}(\mathrm{A}, \mathrm{B}, \mathrm{G}, \mathrm{H}) \in\{\mathrm{d}(\mathrm{Gu}, \mathrm{Hu}), \mathrm{d}(\mathrm{Au}, \mathrm{Gu}), \mathrm{d}(\mathrm{Bu}, \mathrm{Hu}), \mathrm{d}(\mathrm{Au}, \mathrm{Hu}), \mathrm{d}(\mathrm{Bu}, \mathrm{Gu})\}$

$$
=\left\{0, \mathrm{~d}\left(\mathrm{~m}_{1}, \mathrm{~m}_{2}\right)\right\}
$$

Hence $\mathrm{d}\left(\mathrm{m}_{1}, \mathrm{~m}_{2}\right)=0$ i.e. $\mathrm{m}_{1}=\mathrm{m}_{2}$.

If $\{A, G\}$ and $\{B, H\}$ are weakly compatible, then by using Lemma1.18, $\mathrm{u}$ is a unique fixed point of $\mathrm{A}, \mathrm{B}, \mathrm{G}$ and $\mathrm{H}$.

Corollary 2.2. Let X satisfies the same conditions as in Theorem2.1. Suppose the mapping A and B be dominating maps of $\mathrm{X}$ into itself satisfying the condition :

$$
\begin{aligned}
& \forall \mathrm{x}, \mathrm{y} \in \mathrm{X}, \mathrm{d}(\mathrm{Ax}, \mathrm{By}) \leq \mathrm{bN}_{\mathrm{x}, \mathrm{y}}(\mathrm{A}, \mathrm{B}) \\
& \qquad \text { where } \mathrm{b}<\frac{1}{s(s+1)} \text { and }
\end{aligned}
$$

$\mathrm{N}_{\mathrm{x}, \mathrm{y}}(\mathrm{A}, \mathrm{B}) \in\{\mathrm{d}(\mathrm{x}, \mathrm{y}), \mathrm{d}(\mathrm{Ax}, \mathrm{x}), \mathrm{d}(\mathrm{By}, \mathrm{y}), \mathrm{d}(\mathrm{Ax}, \mathrm{y}), \mathrm{d}(\mathrm{y}, \mathrm{Bx})\}$

then $\mathrm{A}$ and $\mathrm{B}$ have a unique common fixed point.

Example 2.3. Let $\mathrm{d}: \mathrm{X} \times \mathrm{X} \rightarrow \mathrm{E}$ where $\mathrm{X}=[0, \infty), \mathrm{E}=\mathrm{C}[0,1]$ defined by

$$
\mathrm{d}(\mathrm{x}, \mathrm{y})(\mathrm{t})=|\mathrm{x}-\mathrm{y}|^{2} \mathrm{e}^{\mathrm{t}}
$$

Then $(X, d, E)$ is E-b-metric space.

We define an ordering $\leqslant$ on $\mathrm{X}$ as $\mathrm{x} \leqslant \mathrm{y} \Leftrightarrow \mathrm{y} \leq \mathrm{x} \forall \mathrm{x}, \mathrm{y} \in \mathrm{X}$

Let $A, B, G, H: X \rightarrow X$ be defined by

$$
\mathrm{Ax}=\frac{5 \mathrm{x}}{7}, \mathrm{Bx}=\frac{3 x}{7}, \mathrm{Gx}=\frac{7 x}{3}, \mathrm{Hx}=\frac{7 x}{5} \forall \mathrm{x} \in \mathrm{X} .
$$

Clearly, $\mathrm{A}(\mathrm{X}) \subseteq \mathrm{H}(\mathrm{X})$ and $\mathrm{B}(\mathrm{X}) \subseteq \mathrm{G}(\mathrm{X})$ and the pairs $(\mathrm{H}, \mathrm{A})$ and $(\mathrm{G}, \mathrm{B})$ partially weakly increasing that is $\mathrm{Hx}=\frac{7 x}{5} \geq \mathrm{x}=\mathrm{AHx}$, which gives $\mathrm{Hx} \leqslant \mathrm{AHx}$ and $\mathrm{Gx}=\frac{7 x}{3} \geq \mathrm{x}=\mathrm{BGx}$, which gives Gx $\leqslant \mathrm{BGx}$. Also, $\mathrm{A}$ and $\mathrm{B}$ are dominating maps, that is, $\mathrm{Ax}=\frac{5 x}{7} \leq \mathrm{x}$ and $\mathrm{Bx}=\frac{3 x}{7} \leq \mathrm{x}$ for all $\mathrm{x} \in \mathrm{X}$ implies that $\mathrm{x} \leqslant$ $A x$ and $x \nLeftarrow B x$ for all $x \in X$. Furthormore, $A$ and $B$ are weak annihilators of $H$ and $G$, respectively, that is, $\mathrm{AHx} \leqslant \mathrm{x}$ and $\mathrm{BGx} \leqslant \mathrm{x}$ for all $\mathrm{x} \in \mathrm{X}$.

For $\mathrm{x}, \mathrm{y} \in \mathrm{X}$,

$$
\mathrm{d}(\text { Ax, By })(\mathrm{t})=\left(\left|\frac{5 x}{7}-\frac{3 y}{7}\right|^{2}\right) \mathrm{e}^{\mathrm{t}}=\frac{1}{49}\left(|5 x-3 y|^{2}\right) \mathrm{e}^{\mathrm{t}}
$$




$$
\begin{aligned}
\mathrm{d}(\mathrm{Gx}, \mathrm{Hy})(\mathrm{t}) & =\left(\left|\frac{7 x}{3}-\frac{7 y}{5}\right|^{2}\right) \mathrm{e}^{\mathrm{t}} \\
\mathrm{d}(\text { Ax, Gx })(\mathrm{t}) & =\left(\left|\frac{5 x}{7}-\frac{7 x}{3}\right|^{2}\right) \mathrm{e}^{\mathrm{t}}=\left(\frac{34 x}{21}\right)^{2} \mathrm{e}^{\mathrm{t}} \\
\mathrm{d}(\text { By, Hy })(\mathrm{t}) & =\left(\left|\frac{3 y}{7}-\frac{7 y}{5}\right|^{2}\right) \mathrm{e}^{\mathrm{t}}=\left(\frac{34 y}{35}\right)^{2} \mathrm{e}^{\mathrm{t}} \\
\mathrm{d}(\text { Ax, Hy) })(\mathrm{t}) & =\left(\left|\frac{5 x}{7}-\frac{7 y}{5}\right|^{2}\right) \mathrm{e}^{\mathrm{t}} \\
\mathrm{d}(\text { By, Gx })(\mathrm{t}) & =\left(\left|\frac{3 y}{7}-\frac{7 x}{5}\right|^{2}\right) \mathrm{e}^{\mathrm{t}} \\
\mathrm{d}(\text { Ax, By })(\mathrm{t}) & =\left(\left|\frac{5 x}{7}-\frac{3 y}{7}\right|^{2}\right) \mathrm{e}^{\mathrm{t}} \\
& \leq\left(\frac{5 x}{7}\right)^{2} \mathrm{e}^{\mathrm{t}} \quad \text { if }\left(\frac{5 x}{7}\right) \geq\left(\frac{3 y}{7}\right) \\
& =b \mathrm{bd}(\mathrm{Ax}, \mathrm{Gx})(\mathrm{t}), \\
&
\end{aligned}
$$

where $d(A x, G x) \in N_{x, y}(A, B, G, H)$

Hence $\mathrm{d}(\mathrm{Ax}, \mathrm{By}) \leq \mathrm{bN}_{\mathrm{x}, \mathrm{y}}(\mathrm{A}, \mathrm{B}, \mathrm{G}, \mathrm{H})$, where $\mathrm{b}=\frac{2}{3}$, is satisfied for $\left(\frac{5 x}{7}\right) \geq\left(\frac{3 y}{7}\right)$

$$
\begin{aligned}
& \mathrm{d}(\text { Ax, By })(\mathrm{t})=\left(\left|\frac{5 x}{7}-\frac{3 y}{7}\right|^{2}\right) \mathrm{e}^{\mathrm{t}} \leq\left(\frac{3 y}{7}\right)^{2} \mathrm{e}^{\mathrm{t}} \quad \text { if }\left(\frac{5 x}{7}\right) \leq\left(\frac{3 y}{7}\right) \\
& \leq \mathrm{b}\left(\frac{34 y}{35}\right)^{2} \mathrm{e}^{\mathrm{t}} \text { with } \mathrm{b}=\frac{2}{3} \\
&=\text { bd(By, Hy })(\mathrm{t}), \text { where } \mathrm{d}(\text { By, Hy }) \in \mathrm{N}_{\mathrm{x}, \mathrm{y}}(\mathrm{A}, \mathrm{B}, \mathrm{G}, \mathrm{H})
\end{aligned}
$$

Hence $\mathrm{d}(\mathrm{Ax}, \mathrm{By}) \leq \mathrm{bN}_{\mathrm{x}, \mathrm{y}}(\mathrm{A}, \mathrm{B}, \mathrm{G}, \mathrm{H}), \forall \mathrm{x}$ and $\mathrm{y}$.

Thus all the conditions of Theorem 2.1 are satisfied with $\mathrm{b}=\frac{2}{3} \in[0,1)$ and 0 is

the unique common fixed point of the mappings $\mathrm{A}, \mathrm{B}, \mathrm{G}$ and $\mathrm{H}$.

Theorem 2.4. Let $\mathrm{X}$ be an $\mathrm{E}-\mathrm{b}-$-metric space with $\mathrm{E}$ is Archimedean. Suppose the mapping A, B, G and $\mathrm{H}$ be self-maps on $X$, $(H, A)$ and $(G, B)$ partially weakly increasing with $A(X) \subseteq H(X)$ and $B(X) \subseteq G(X)$. Further the dominating maps A and B are weak annihilators of $\mathrm{H}$ and $\mathrm{G}$, respectively. Suppose the four mappings A, $\mathrm{B}, \mathrm{G}, \mathrm{H}: \mathrm{X} \rightarrow \mathrm{X}$ satisfy the following condition :

For all $\mathrm{x}, \mathrm{y} \in \mathrm{X}$,

$$
\begin{aligned}
& \mathrm{d}(\mathrm{Ax}, \mathrm{By}) \leq \mathrm{bN}_{\mathrm{x}, \mathrm{y}}(\mathrm{A}, \mathrm{B}, \mathrm{G}, \mathrm{H}) \\
& \text { where } \mathrm{b}<\frac{2}{\left(\mathrm{~s}^{2}+\mathrm{s}+1\right)} \text { and }
\end{aligned}
$$


$\mathrm{N}_{\mathrm{x}, \mathrm{y}}(\mathrm{A}, \mathrm{B}, \mathrm{G}, \mathrm{H}) \in\left\{\frac{1}{2}[\mathrm{~d}(\mathrm{Gx}, \mathrm{Hy})+\mathrm{d}(\mathrm{Ax}, \mathrm{Gx})], \frac{1}{2}[\mathrm{~d}(\mathrm{Gx}, \mathrm{Hy})+\mathrm{d}(\mathrm{By}, \mathrm{Hy})], \frac{1}{2}[\mathrm{~d}(\mathrm{Gx}, \mathrm{Hy})+\right.$ $\mathrm{d}(\mathrm{Ax}, \mathrm{Hy})], \frac{1}{2}[\mathrm{~d}(\mathrm{Gx}, \mathrm{Hy})+\mathrm{d}(\mathrm{By}, \mathrm{Gx})], \frac{1}{2}[\mathrm{~d}(\mathrm{By}, \mathrm{Hy})+\mathrm{d}(\mathrm{Ax}, \mathrm{Hy})], \frac{1}{2}[\mathrm{~d}(\mathrm{Ax}, \mathrm{Hy})+$ $\left.\mathrm{d}(\mathrm{By}, \mathrm{Gx})], \frac{1}{2}[\mathrm{~d}(\mathrm{By}, \mathrm{Hy})+\mathrm{d}(\mathrm{By}, \mathrm{Gx})]\right\}$.

If for a nondecreasing sequence $\left\{\mathrm{x}_{\mathrm{n}}\right\}$ with $\mathrm{x}_{\mathrm{n}} \leq \mathrm{y}_{\mathrm{n}}$ for all $\mathrm{n}$ and $\mathrm{y}_{\mathrm{n}} \stackrel{d . E}{\longrightarrow} m$ implies that $x_{n} \leq m$, the pairs $\{A, G\}$ and $\{B, H\}$ are weakly compatible and one of $A(X), B(X), G(X)$ and $H(X)$ is $E-$ complete then $\mathrm{A}, \mathrm{B}, \mathrm{G}$ and $\mathrm{H}$ have a common fixed point.

Proof : We define the sequence $\left\{\mathrm{x}_{\mathrm{n}}\right\}$ and $\left\{\mathrm{y}_{\mathrm{n}}\right\}$ as in proof of theorem 2.1

Firstly, show that

$$
\mathrm{d}\left(\mathrm{y}_{2 \mathrm{n}+1}, \mathrm{y}_{2 \mathrm{n}+2}\right) \leq \delta \mathrm{d}\left(\mathrm{y}_{2 \mathrm{n}}, \mathrm{y}_{2 \mathrm{n}+1}\right) \text { for all } \mathrm{n}, \text { where } \delta<1
$$

From (5), we have

$$
d\left(y_{2 n+1}, y_{2 n+2}\right)=d\left(A_{2 n}, B_{2 n+1}\right) \leq b N_{x_{2 n}, x_{2 n+1}}(A, B, G, H) \text { for } n=0,1,2,3 \ldots \ldots
$$

Since

$$
\begin{aligned}
& \mathrm{N}_{\mathrm{x}_{2 n}, \mathrm{x}_{2 n+1}}(A, B, G, H) \in\left\{\frac{1}{2}\left[\mathrm{~d}\left(\mathrm{Gx}_{2 \mathrm{n}}, \mathrm{Hx}_{2 \mathrm{n}+1}\right)+\mathrm{d}\left(\mathrm{Ax}_{2 \mathrm{n}}, \mathrm{Gx}_{2 \mathrm{n}}\right)\right], \frac{1}{2}\left[\mathrm{~d}\left(\mathrm{Gx}_{2 \mathrm{n}}, \mathrm{Hx}_{2 \mathrm{n}+1}\right)+\right.\right. \\
& \left.\mathrm{d}\left(\mathrm{Bx}_{2 \mathrm{n}+1}, \mathrm{Hx}_{2 \mathrm{n}+1}\right)\right], \frac{1}{2}\left[\mathrm{~d}\left(\mathrm{Gx}_{2 \mathrm{n}}, \mathrm{Hx}_{2 \mathrm{n}+1}\right)+\mathrm{d}\left(\mathrm{Ax}_{2 \mathrm{n}}, \mathrm{Hx}_{2 \mathrm{n}+1}\right)\right], \frac{1}{2}\left[\mathrm{~d}\left(\mathrm{Gx}_{2 \mathrm{n}}, \mathrm{Hx}_{2 \mathrm{n}+1}\right)+\mathrm{d}\left(\mathrm{Bx}_{2 \mathrm{n}+1}, \mathrm{Gx}_{2 \mathrm{n}}\right)\right], \frac{1}{2}\left[\mathrm { d } \left(\mathrm{Bx}_{2 \mathrm{n}+1},\right.\right. \\
& \left.\left.\left.H x_{2 n+1}\right)+d\left(A x_{2 n}, H x_{2 n+1}\right)\right], \frac{1}{2}\left[d\left(A_{2 n}, H x_{2 n+1}\right)+d\left(B x_{2 n+1}, G x_{2 n}\right)\right], \frac{1}{2}\left[d\left(B_{2 n+1}, H_{2 n+1}\right)+d\left(B_{2 n+1}, G_{2 n}\right)\right]\right\} \\
& =\left\{\frac{1}{2}\left[d\left(y_{2 n}, y_{2 n+1}\right)+d\left(y_{2 n+1}, y_{2 n}\right)\right], \frac{1}{2}\left[d\left(y_{2 n}, y_{2 n+1}\right)+d\left(y_{2 n+2}, y_{2 n+1}\right)\right], \frac{1}{2}\left[d\left(y_{2 n}, y_{2 n+1}\right)+\right.\right. \\
& \left.\mathrm{d}\left(\mathrm{y}_{2 \mathrm{n}+1}, \mathrm{y}_{2 \mathrm{n}+1}\right)\right], \frac{1}{2}\left[\mathrm{~d}\left(\mathrm{y}_{2 \mathrm{n}}, \mathrm{y}_{2 \mathrm{n}+1}\right)+\mathrm{d}\left(\mathrm{y}_{2 \mathrm{n}+2}, \mathrm{y}_{2 \mathrm{n}}\right)\right], \frac{1}{2}\left[\mathrm{~d}\left(\mathrm{y}_{2 \mathrm{n}+2}, \mathrm{y}_{2 \mathrm{n}+1}\right)+\mathrm{d}\left(\mathrm{y}_{2 \mathrm{n}+1}, \mathrm{y}_{2 \mathrm{n}+1}\right)\right], \frac{1}{2}\left[\mathrm{~d}\left(\mathrm{y}_{2 \mathrm{n}+1}, \mathrm{y}_{2 \mathrm{n}+1}\right)+\right. \\
& \left.\mathrm{d}\left(\mathrm{y}_{2 \mathrm{n}+2}, \mathrm{y}_{2 \mathrm{n}}\right)\right], \frac{1}{2}\left[\mathrm{~d}\left(\mathrm{y}_{2 \mathrm{n}+2}, \mathrm{y}_{2 \mathrm{n}+1}\right)+\mathrm{d}\left(\mathrm{y}_{2 \mathrm{n}+1}, \mathrm{y}_{2 \mathrm{n}+1}\right)\right], \frac{1}{2}\left[\mathrm{~d}\left(\mathrm{y}_{2 \mathrm{n}+1}, \mathrm{y}_{2 \mathrm{n}+1}\right)+\mathrm{d}\left(\mathrm{y}_{2 \mathrm{n}+2}, \mathrm{y}_{2 \mathrm{n}}\right)\right], \frac{1}{2}\left[\mathrm{~d}\left(\mathrm{y}_{2 \mathrm{n}+2}, \mathrm{y}_{2 \mathrm{n}+1}\right)+\mathrm{d}\left(\mathrm{y}_{2 \mathrm{n}+2},\right.\right. \\
& \mathrm{y}_{2 \mathrm{n}} \text { ] } \\
& =\left\{\left[d\left(y_{2 n}, y_{2 n+1}\right)\right], \frac{1}{2}\left[d\left(y_{2 n}, y_{2 n+1}\right)+d\left(y_{2 n+2}, y_{2 n+1}\right)\right], \frac{1}{2}\left[d\left(y_{2 n}, y_{2 n+1}\right)\right], \frac{1}{2}\left[d\left(y_{2 n}, y_{2 n+1}\right)+\right.\right. \\
& \left.\left.\mathrm{d}\left(\mathrm{y}_{2 \mathrm{n}+2}, \mathrm{y}_{2 \mathrm{n}}\right)\right], \frac{1}{2}\left[\mathrm{~d}\left(\mathrm{y}_{2 \mathrm{n}+2}, \mathrm{y}_{2 \mathrm{n}+1}\right)\right], \frac{1}{2}\left[\mathrm{~d}\left(\mathrm{y}_{2 \mathrm{n}+2}, \mathrm{y}_{2 \mathrm{n}}\right)\right], \frac{1}{2}\left[\left(\mathrm{y}_{2 \mathrm{n}+2}, \mathrm{y}_{2 \mathrm{n}+1}\right)+\mathrm{d}\left(\mathrm{y}_{2 \mathrm{n}+2}, \mathrm{y}_{2 \mathrm{n}}\right)\right]\right\}
\end{aligned}
$$

Let us suppose that if $\mathrm{N}_{\mathrm{x}_{2 n}, \mathrm{x}_{2 n+1}}(A, B, G, H)=\frac{1}{2}\left[\mathrm{~d}\left(\mathrm{y}_{2 \mathrm{n}}, \mathrm{y}_{2 \mathrm{n}+1}\right)+\mathrm{d}\left(\mathrm{y}_{2 \mathrm{n}+2}, \mathrm{y}_{2 \mathrm{n}+1}\right)\right]$,

$$
\begin{aligned}
\text { Then } \mathrm{d}\left(\mathrm{y}_{2 \mathrm{n}+1}, \mathrm{y}_{2 \mathrm{n}+2}\right) & \leq \frac{\mathrm{b}}{2}\left[\mathrm{~d}\left(\mathrm{y}_{2 \mathrm{n}}, \mathrm{y}_{2 \mathrm{n}+1}\right)\right]+\frac{\mathrm{b}}{2}\left[\mathrm{~d}\left(\mathrm{y}_{2 \mathrm{n}+2}, \mathrm{y}_{2 \mathrm{n}+1}\right)\right] \\
\left(1-\frac{b}{2}\right) \mathrm{d}\left(\mathrm{y}_{2 \mathrm{n}+1}, \mathrm{y}_{2 \mathrm{n}+2}\right) & \leq \frac{\mathrm{b}}{2}\left[\mathrm{~d}\left(\mathrm{y}_{2 \mathrm{n}}, \mathrm{y}_{2 \mathrm{n}+1}\right)\right]
\end{aligned}
$$




$$
\mathrm{d}\left(\mathrm{y}_{2 \mathrm{n}+1}, \mathrm{y}_{2 \mathrm{n}+2}\right) \leq\left(\frac{\frac{b}{2}}{1-\frac{b}{2}}\right)\left[\mathrm{d}\left(\mathrm{y}_{2 \mathrm{n}}, \mathrm{y}_{2 \mathrm{n}+1}\right)\right] \leq \delta_{1}\left[\mathrm{~d}\left(\mathrm{y}_{2 \mathrm{n}}, \mathrm{y}_{2 \mathrm{n}+1}\right)\right] \quad \text { where } \delta_{1}=\left(\frac{\frac{b}{2}}{1-\frac{b}{2}}\right)
$$

If $\mathrm{N}_{\mathrm{x}_{2 \mathrm{n}}, \mathrm{x}_{2 \mathrm{n}+1}}(\mathrm{~A}, \mathrm{~B}, \mathrm{G}, \mathrm{H})=\frac{1}{2}\left[\mathrm{~d}\left(\mathrm{y}_{2 \mathrm{n}}, \mathrm{y}_{2 \mathrm{n}+1}\right)+\mathrm{d}\left(\mathrm{y}_{2 \mathrm{n}+2}, \mathrm{y}_{2 \mathrm{n}}\right)\right]$

then $d\left(y_{2 n+1}, y_{2 n+2}\right) \leq \frac{b}{2}\left[d\left(y_{2 n}, y_{2 n+1}\right)\right]+\frac{b}{2}\left[\operatorname{sd}\left(y_{2 n+2}, y_{2 n+1}\right)+\operatorname{sd}\left(y_{2 n+1}, y_{2 n}\right)\right]$

$$
\begin{aligned}
&\left(1-\frac{\mathrm{sb}}{2}\right) \mathrm{d}\left(\mathrm{y}_{2 \mathrm{n}+1}, \mathrm{y}_{2 \mathrm{n}+2}\right) \leq(1+s) \frac{b}{2}\left[\mathrm{~d}\left(\mathrm{y}_{2 \mathrm{n}}, \mathrm{y}_{2 \mathrm{n}+1}\right)\right] \\
& \mathrm{d}\left(\mathrm{y}_{2 \mathrm{n}+1}, \mathrm{y}_{2 \mathrm{n}+2}\right) \leq \frac{b}{2}\left(\frac{1+s}{1-\frac{s b}{2}}\right)\left[\mathrm{d}\left(\mathrm{y}_{2 \mathrm{n}}, \mathrm{y}_{2 \mathrm{n}+1}\right)\right] \leq \delta_{2}\left[\mathrm{~d}\left(\mathrm{y}_{2 \mathrm{n}}, \mathrm{y}_{2 \mathrm{n}+1}\right)\right], \\
& \text { where } \delta_{2}=\frac{b}{2}\left(\frac{1+s}{1-\frac{s b}{2}}\right)
\end{aligned}
$$

If $\mathrm{N}_{\mathrm{x}_{2 \mathrm{n}}, \mathrm{x}_{2 \mathrm{n}+1}}(\mathrm{~A}, \mathrm{~B}, \mathrm{G}, \mathrm{H})=\frac{1}{2}\left[\mathrm{~d}\left(\mathrm{y}_{2 \mathrm{n}}, \mathrm{y}_{2 \mathrm{n}+2}\right)\right]$

$$
\text { then } d\left(y_{2 n+1}, y_{2 n+2}\right) \leq \frac{b}{2}\left[\operatorname{sd}\left(y_{2 n}, y_{2 n+1}\right)+\operatorname{sd}\left(y_{2 n+1}, y_{2 n+2}\right)\right]
$$

$$
\begin{array}{r}
\mathrm{d}\left(\mathrm{y}_{2 \mathrm{n}+1}, \mathrm{y}_{2 \mathrm{n}+2}\right) \leq\left(\frac{\frac{s b}{2}}{1-\frac{s b}{2}}\right)\left[\mathrm{d}\left(\mathrm{y}_{2 \mathrm{n}}, \mathrm{y}_{2 \mathrm{n}+1}\right)\right] \leq \delta_{3}\left[\mathrm{~d}\left(\mathrm{y}_{2 \mathrm{n}}, \mathrm{y}_{2 \mathrm{n}+1}\right)\right], \\
\text { where } \delta_{3}=\left(\frac{\frac{s b}{2}}{1-\frac{s b}{2}}\right)
\end{array}
$$

Finally suppose that, $\mathrm{N}_{\mathrm{x}_{2 n}, \mathrm{x}_{2 n+1}}(\mathrm{~A}, \mathrm{~B}, \mathrm{G}, \mathrm{H})=\frac{1}{2}\left[\mathrm{~d}\left(\mathrm{y}_{2 \mathrm{n}+2}, \mathrm{y}_{2 \mathrm{n}+1}\right)+\mathrm{d}\left(\mathrm{y}_{2 \mathrm{n}+2}, \mathrm{y}_{2 \mathrm{n}}\right)\right]$

Then $d\left(y_{2 n+1}, y_{2 n+2}\right) \leq \frac{b}{2}\left[d\left(y_{2 n+2}, y_{2 n+1}\right)\right]+\frac{b}{2}\left[\operatorname{sd}\left(y_{2 n+2}, y_{2 n+1}\right)+\operatorname{sd}\left(y_{2 n+1}, y_{2 n}\right)\right]$

$$
\begin{aligned}
& \left(1-\frac{\mathrm{b}}{2}-\frac{\mathrm{sb}}{2}\right) \mathrm{d}\left(\mathrm{y}_{2 \mathrm{n}+1}, \mathrm{y}_{2 \mathrm{n}+2}\right) \leq \frac{\mathrm{sb}}{2} \mathrm{~d}\left(\mathrm{y}_{2 \mathrm{n}+1}, \mathrm{y}_{2 \mathrm{n}}\right) \\
& \mathrm{d}\left(\mathrm{y}_{2 \mathrm{n}+1}, \mathrm{y}_{2 \mathrm{n}+2}\right) \leq\left(\frac{\frac{\mathrm{sb}}{2}}{1-\frac{\mathrm{b}}{2}-\frac{s \mathrm{~b}}{2}}\right)=\mathrm{d}\left(\mathrm{y}_{2 \mathrm{n}+1}, \mathrm{y}_{2 \mathrm{n}}\right) \leq \delta_{4} \mathrm{~d}\left(\mathrm{y}_{2 \mathrm{n}+1}, \mathrm{y}_{2 \mathrm{n}}\right), \\
& \text { where } \delta_{4}=\left(\frac{\frac{s b}{2}}{1-\frac{b}{2}-\frac{s b}{2}}\right)
\end{aligned}
$$


Let $\delta=\left\{\delta_{1}, \delta_{2}, \delta_{3}, \delta_{4}\right\}$ then $\mathrm{d}\left(\mathrm{y}_{2 \mathrm{n}+1}, \mathrm{y}_{2 \mathrm{n}+2}\right) \leq \delta \mathrm{d}\left(\mathrm{y}_{2 \mathrm{n}+1}, \mathrm{y}_{2 \mathrm{n}}\right)$.

Similarly $d\left(\mathrm{y}_{2 \mathrm{n}+2}, \mathrm{y}_{2 \mathrm{n}+3}\right) \leq \delta \mathrm{d}\left(\mathrm{y}_{2 \mathrm{n}+1}, \mathrm{y}_{2 \mathrm{n}+2}\right)$

Therefore, $\quad \mathrm{d}\left(\mathrm{y}_{\mathrm{n}}, \mathrm{y}_{\mathrm{n}+1}\right) \leq \delta \mathrm{d}\left(\mathrm{y}_{\mathrm{n}-1}, \mathrm{y}_{\mathrm{n}}\right)$

Thus $\mathrm{d}\left(\mathrm{y}_{\mathrm{n}}, \mathrm{y}_{\mathrm{n}+1}\right) \leq(\delta)^{\mathrm{n}} \mathrm{d}\left(\mathrm{y}_{0}, \mathrm{y}_{1}\right)$,

By using (8), $\forall \mathrm{n}$ and $\mathrm{p}$, we have

$$
\begin{aligned}
\mathrm{d}\left(\mathrm{y}_{\mathrm{n}}, \mathrm{y}_{\mathrm{n}+\mathrm{p}}\right) & \leq \mathrm{sd}\left(\mathrm{y}_{\mathrm{n}}, \mathrm{y}_{\mathrm{n}+1}\right)+\mathrm{s}^{2} \mathrm{~d}\left(\mathrm{y}_{\mathrm{n}+1}, \mathrm{y}_{\mathrm{n}+2}\right)+\ldots \ldots \ldots . .+\mathrm{s}^{\mathrm{p}} \mathrm{d}\left(\mathrm{y}_{\mathrm{n}+\mathrm{p}-1}, \mathrm{y}_{\mathrm{n}+\mathrm{p}}\right) \\
& \leq \mathrm{s}(\delta)^{\mathrm{n}} \mathrm{d}\left(\mathrm{y}_{0}, \mathrm{y}_{1}\right)+\mathrm{s}^{2}(\delta)^{\mathrm{n}+1} \mathrm{~d}\left(\mathrm{y}_{0}, \mathrm{y}_{1}\right)+\ldots \ldots \ldots \ldots+\mathrm{s}^{\mathrm{n}+\mathrm{p}}(\delta)^{n+\mathrm{p}-1} \mathrm{~d}\left(\mathrm{y}_{0}, \mathrm{y}_{1}\right) \\
= & s(\delta)^{n}\left(\frac{1-(s \delta)^{p}}{1-(s \delta)}\right) \mathrm{d}\left(\mathrm{y}_{0}, \mathrm{y}_{1}\right) \leq\left(\frac{s(\delta)^{n}}{1-s \delta}\right) \mathrm{d}\left(\mathrm{y}_{0}, \mathrm{y}_{1}\right)
\end{aligned}
$$

It is given that $\mathrm{E}$ is Archimedean, then $\left(\mathrm{y}_{\mathrm{n}}\right)$ is an $\mathrm{E}-$ Cauchy sequence. Let us suppose that $\mathrm{G}(\mathrm{X})$ is E-complete, so there exists $\mathrm{u} \in \mathrm{G}(\mathrm{X})$ such that

$$
\mathrm{Gx}_{2 \mathrm{n}}=\mathrm{y}_{2 \mathrm{n}} \stackrel{\text { d.E. }}{\longrightarrow} \mathrm{u}
$$

Thus there exists a sequence $\left\{\mathrm{e}_{\mathrm{n}}\right\}$ in $\mathrm{E}$ such that $\mathrm{e}_{\mathrm{n}} \downarrow 0$ and $\mathrm{d}\left(\mathrm{Gx}_{2 \mathrm{n}}, \mathrm{u}\right) \leq \mathrm{e}_{\mathrm{n}}$. On the other hand we can find out $v$ in $X$ such that $G v=u$. Now we are going to prove that $A v=u$. Since $x_{2 n+1} \leq B x_{2 n+1}$ and

$$
\mathrm{Bx}_{2 \mathrm{n}+1} \stackrel{\text { d.E. }}{\longrightarrow} \mathrm{u} \text { which implies that } \mathrm{x}_{2 \mathrm{n}+1} \leq \mathrm{u} \text { and } \mathrm{u} \leq \mathrm{Bu}=
$$

$\mathrm{BGv} \leq \mathrm{v}$ which implies that $\mathrm{x}_{2 \mathrm{n}+1} \leq \mathrm{v}$.

Consider

$\mathrm{d}(\mathrm{Av}, \mathrm{u}) \leq \operatorname{sd}\left(\mathrm{Av}, \mathrm{Bx}_{2 \mathrm{n}+1}\right)+\operatorname{sd}\left(\mathrm{Bx}_{2 \mathrm{n}+1}, \mathrm{u}\right) \leq \operatorname{sbN}_{\mathrm{V}, \mathrm{X}_{2 n+1}}(\mathrm{~A}, \mathrm{~B}, \mathrm{G}, \mathrm{H})+\mathrm{se}_{\mathrm{n}+1}$

where $\mathrm{bN}_{\mathrm{V}, \mathrm{X}_{2 n+1}}(\mathrm{~A}, \mathrm{~B}, \mathrm{G}, \mathrm{H}) \in\left\{\frac{1}{2}\left[\mathrm{~d}\left(\mathrm{Gv}, \mathrm{Hx}_{2 \mathrm{n}+1}\right)+\mathrm{d}(\mathrm{Av}, \mathrm{Gv})\right], \frac{1}{2}\left[\mathrm{~d}\left(\mathrm{Gv}, \mathrm{Hx}_{2 \mathrm{n}+1}\right)+\right.\right.$

$$
\begin{aligned}
& \left.\mathrm{d}\left(\mathrm{Bx}_{2 \mathrm{n}+1}, H \mathrm{Hx}_{2 \mathrm{n}+1}\right)\right], \frac{1}{2}\left[\mathrm{~d}\left(\mathrm{Gv}, \mathrm{Hx}_{2 \mathrm{n}+1}\right)+\mathrm{d}\left(\mathrm{Av}, \mathrm{Hx}_{2 \mathrm{n}+1}\right)\right], \frac{1}{2}\left[\mathrm{~d}\left(\mathrm{Gv}, \mathrm{Hx}_{2 \mathrm{n}+1}\right)+\mathrm{d}\left(\mathrm{Bx}_{2 \mathrm{n}+1}, \mathrm{Gv}\right)\right], \\
& \frac{1}{2}\left[\mathrm{~d}\left(\mathrm{Bx}_{2 \mathrm{n}+1}, \mathrm{Hx}_{2 \mathrm{n}+1}\right)+\mathrm{d}\left(\mathrm{Av}, \mathrm{Hx}_{2 \mathrm{n}+1}\right)\right], \frac{1}{2}\left[\mathrm{~d}\left(\mathrm{Av}, \mathrm{Hx}_{2 \mathrm{n}+1}\right)+\mathrm{d}\left(\mathrm{Bx}_{2 \mathrm{n}+1}, \mathrm{Gv}\right)\right], \frac{1}{2}\left[\mathrm{~d}\left(\mathrm{Bx}_{2 \mathrm{n}+1}, \mathrm{Hx}_{2 \mathrm{n}+1}\right)+\mathrm{d}\left(\mathrm{Bx}_{2 \mathrm{n}+1},\right.\right. \\
& \mathrm{Gv})]\} \forall \mathrm{n} .
\end{aligned}
$$

There are seven possibilities:

Case $1: \mathrm{d}(\mathrm{Av}, \mathrm{u}) \leq \frac{\mathrm{sb}}{2}\left[\mathrm{~d}\left(\mathrm{Gv}, \mathrm{Hx}_{2 \mathrm{n}+1}\right)+\mathrm{d}(\mathrm{Av}, \mathrm{Gv})\right]+\mathrm{se}_{\mathrm{n}+1}$

$$
\begin{aligned}
& \mathrm{d}(\mathrm{Av}, \mathrm{u}) \leq \frac{\mathrm{sb}}{2}\left[\mathrm{~d}\left(\mathrm{u}, \mathrm{Hx}_{2 \mathrm{n}+1}\right)+\mathrm{d}(\mathrm{Av}, \mathrm{u})\right]+\mathrm{se}_{\mathrm{n}+1} \\
&\left(1-\frac{s b}{2}\right) \mathrm{d}(\mathrm{Av}, \mathrm{u}) \leq \frac{\mathrm{sb}}{2} \mathrm{e}_{\mathrm{n}+1}+\mathrm{se}_{\mathrm{n}+1} \\
& \mathrm{~d}(\mathrm{Av}, \mathrm{u}) \leq \frac{\mathrm{s}\left(\frac{\mathrm{b}}{2}+1\right)}{\left(1-\frac{s b}{2}\right)} \mathrm{e}_{\mathrm{n}}
\end{aligned}
$$

Case $2: d(A v, u) \leq \frac{s b}{2}\left[d\left(G v, H_{2 n+1}\right)+d\left(B x_{2 n+1}, H x_{2 n+1}\right)\right]+s_{n+1}$

$$
\begin{aligned}
& \leq \frac{s b}{2}\left[\mathrm{~d}\left(\mathrm{u}, \mathrm{Hx}_{2 \mathrm{n}+1}\right)+\mathrm{d}\left(\mathrm{Bx}_{2 \mathrm{n}+1}, \mathrm{Hx}_{2 \mathrm{n}+1}\right)\right]+\mathrm{se}_{\mathrm{n}+1} \\
& \leq \frac{\mathrm{sb}}{2}\left[\mathrm{~d}\left(\mathrm{u}, \mathrm{Hx}_{2 \mathrm{n}+1}\right)+\mathrm{sd}\left(\mathrm{Bx}_{2 \mathrm{n}+1}, \mathrm{u}\right)+\mathrm{sd}\left(\mathrm{u}, \mathrm{Hx}_{2 \mathrm{n}+1}\right)\right]+\mathrm{se}_{\mathrm{n}+1} \\
& \leq \frac{s b}{2} \mathrm{e}_{\mathrm{n}+1}+\frac{s^{2} b}{2} \mathrm{e}_{\mathrm{n}+1}+\frac{s^{2} b}{2} \mathrm{e}_{\mathrm{n}+1}+\mathrm{se}_{\mathrm{n}+1}
\end{aligned}
$$




$$
\leq \mathrm{s}\left(\frac{b}{2}+s b+1\right) \mathrm{e}_{\mathrm{n}}
$$

Case $3: d(A v, u) \leq \frac{s b}{2}\left[d\left(G v, H x_{2 n+1}\right)+d\left(A v, H x_{2 n+1}\right)\right]+s_{n+1}$

$$
d(A v, u) \leq \frac{s b}{2}\left[d\left(u, H_{2 n+1}\right)+\operatorname{sd}(A v, u)+\operatorname{sd}\left(u, H_{2 n+1}\right)\right]+s_{n+1}
$$

$$
\left(1-\frac{s^{2} b}{2}\right) \mathrm{d}(\mathrm{Av}, \mathrm{u}) \leq \frac{\mathrm{sb}}{2} \mathrm{e}_{\mathrm{n}+1}+\frac{s^{2} b}{2} \mathrm{e}_{\mathrm{n}+1}+\mathrm{se}_{\mathrm{n}+1}
$$

$$
\mathrm{d}(\mathrm{Av}, \mathrm{u}) \leq \mathrm{s}\left(\frac{1+\frac{b}{2}+\frac{s b}{2}}{1-\frac{s^{2} b}{2}}\right) \mathrm{e}_{\mathrm{n}}
$$

Case $4: d(A v, u) \leq \frac{s b}{2}\left[d\left(G v, H x_{2 n+1}\right)+d\left(B_{2 n+1}, G v\right)\right]+s_{n+1}$

$$
\begin{aligned}
& \leq \frac{s b}{2}\left[d\left(u, H_{2 n+1}\right)+d\left(B_{2 n+1}, u\right)+e_{n+1}\right. \\
& \leq \frac{s b}{2} e_{n+1}+\frac{s b}{2} e_{n+1}+s e_{n+1} \leq s(b+1) e_{n}
\end{aligned}
$$

Case $5: d(A v, u) \leq \frac{s b}{2}\left[d\left(B_{2 n+1}, H_{2 n+1}\right)+d\left(A v, H_{2 n+1}\right)\right]+e_{n+1}$

$$
\begin{gathered}
\leq \frac{\mathrm{sb}}{2}\left[\operatorname{sd}_{\left(\mathrm{Bx}_{2 \mathrm{n}+1}, \mathrm{u}\right)+} \mathrm{sd}\left(\mathrm{u}, \mathrm{Hx}_{2 \mathrm{n}+1}\right)\right]+\frac{\mathrm{sb}}{2}\left[\operatorname{sd}(\mathrm{Av}, \mathrm{u})+\mathrm{sd}\left(\mathrm{u}, \mathrm{Hx}_{2 \mathrm{n}+1}\right)\right]+\mathrm{se}_{\mathrm{n}+1}\left(1-\frac{s^{2} b}{2}\right) \mathrm{d}(\mathrm{Av}, \mathrm{u}) \\
\leq \frac{s^{2} b}{2} \mathrm{e}_{\mathrm{n}+1}+\frac{s^{2} b}{2} \mathrm{e}_{\mathrm{n}+1}+\frac{s^{2} b}{2} \mathrm{e}_{\mathrm{n}+1}+\mathrm{se}_{\mathrm{n}+1} \\
\left(1-\frac{s^{2} b}{2}\right) \mathrm{d}(\mathrm{Av}, \mathrm{u}) \leq \frac{3}{2} s^{2} b \mathrm{e}_{\mathrm{n}+1}+\mathrm{se}_{\mathrm{n}+1} \\
\mathrm{~d}(\mathrm{Av}, \mathrm{u}) \leq S\left(\frac{\frac{3}{2} \mathrm{sb}+1}{1-\frac{\mathrm{s}^{2} b}{2}}\right) \mathrm{e}_{\mathrm{n}}
\end{gathered}
$$

Case $6: d(A v, u) \leq \frac{s b}{2}\left[d\left(A v, H_{2 n+1}\right)+d\left(B x_{2 n+1}, G v\right)\right]+s_{n+1}$

$$
\begin{aligned}
& \leq \frac{s b}{2}\left[\operatorname{sd}(\operatorname{Av}, \mathrm{u})+\operatorname{sd}\left(\mathrm{u}, \mathrm{Hx}_{2 \mathrm{n}+1}\right)\right]+\frac{\mathrm{sb}}{2} \mathrm{~d}\left(\mathrm{Bx}_{2 \mathrm{n}+1}, \mathrm{u}\right)+\mathrm{se}_{\mathrm{n}+1} \\
\left(1-\frac{s^{2} b}{2}\right) \mathrm{d}(\mathrm{Av}, \mathrm{u}) & \leq \frac{s^{2} b}{2} \mathrm{e}_{\mathrm{n}+1}+\frac{s b}{2} \mathrm{e}_{\mathrm{n}+1}+\mathrm{se}_{\mathrm{n}+1} \\
\mathrm{~d}(\mathrm{Av}, \mathrm{u}) & \leq \mathrm{s}\left(\frac{1+\frac{b}{2}+\frac{s b}{2}}{1-\frac{s^{2} b}{2}}\right) \mathrm{e}_{\mathrm{n}}
\end{aligned}
$$

Case $7: d(A v, u) \leq \frac{s b}{2}\left[d\left(B x_{2 n+1}, H_{2 n+1}\right)+d\left(B_{2 n+1}, G v\right)\right]+s_{n+1}$ 


$$
\begin{aligned}
& \quad \leq \frac{s b}{2}\left[\operatorname{sd}\left(B_{2 n+1}, u\right)+s d\left(u, H_{2 n+1}\right)\right]+\frac{s b}{2}\left[d\left(B_{2 n+1}, u\right)\right]+e_{n+1} \\
& \leq \frac{s^{2} b}{2} e_{n+1}+\frac{s^{2} b}{2} e_{n+1}+\frac{s b}{2} e_{n+1}+s_{n+1} \\
& \leq s\left(\frac{b}{2}+s b+1\right) \mathrm{e}_{n}
\end{aligned}
$$

It is given that g.l.b. of sequences on the right hand side are zero, then $d(A v, u)=0$, that is $A v=u$. Therefore $A v=G v=u$

Since $\mathrm{u} \in \mathrm{A}(\mathrm{X}) \subseteq \mathrm{H}(\mathrm{X})$, there exists $\mathrm{w} \in \mathrm{X}$ such that $\mathrm{Hw}=\mathrm{u}$.

Now, we show that $\mathrm{Bw}=\mathrm{u}$. As $\mathrm{x}_{2 \mathrm{n}} \leq \mathrm{Ax}_{2 \mathrm{n}}$ and $\mathrm{Ax}_{2 \mathrm{n}} \stackrel{\text { d.E. }}{\longrightarrow} \mathrm{u}$ which implies that $\mathrm{x}_{2 \mathrm{n}} \leq \mathrm{u}$ and $\mathrm{u} \leq \mathrm{Au}=\mathrm{AHw} \leq \mathrm{w}$ which implies that $\mathrm{x}_{2 \mathrm{n}} \leq \mathrm{w}$.

Consider

$$
d(u, B w) \leq \operatorname{sd}\left(u, A x_{2 n}\right)+\operatorname{sd}\left(A_{2 n}, B w\right) \leq \operatorname{se}_{n}+\operatorname{sbN}_{X_{2 n, w}}(A, B, G, H)
$$

where

$$
\begin{gathered}
\mathrm{N}_{\mathrm{X}_{2 \mathrm{n}, \mathrm{w}}}(\mathrm{A}, \mathrm{B}, \mathrm{G}, \mathrm{H}) \in\left\{\frac{1}{2}\left[\mathrm{~d}\left(\mathrm{Gx}_{2 \mathrm{n}}, \mathrm{Hw}\right)+\mathrm{d}\left(\mathrm{Ax}_{2 \mathrm{n}}, \mathrm{Gx}_{2 \mathrm{n}}\right)\right], \frac{1}{2}\left[\mathrm{~d}\left(\mathrm{Gx}_{2 \mathrm{n}}, \mathrm{Hw}\right)+\mathrm{d}(\mathrm{Bw}, \mathrm{Hw})\right],\right. \\
\frac{1}{2}\left[\mathrm{~d}\left(\mathrm{Gx}_{2 \mathrm{n}}, \mathrm{Hw}\right)+\mathrm{d}\left(\mathrm{Ax}_{2 \mathrm{n}}, \mathrm{Hw}\right)\right], \frac{1}{2}\left[\mathrm{~d}\left(\mathrm{Gx}_{2 \mathrm{n}}, \mathrm{Hw}\right)+\mathrm{d}\left(\mathrm{Bw}, \mathrm{Gx}_{2 \mathrm{n}}\right)\right], \frac{1}{2}[\mathrm{~d}(\mathrm{Bw}, \mathrm{Hw})+ \\
\left.\left.\mathrm{d}\left(\mathrm{Ax}_{2 \mathrm{n}}, \mathrm{Hw}\right)\right], \frac{1}{2}\left[\mathrm{~d}\left(\mathrm{Ax}_{2 \mathrm{n}}, \mathrm{Hw}\right)+\mathrm{d}\left(\mathrm{Bw}, \mathrm{Gx}_{2 \mathrm{n}}\right)\right], \frac{1}{2}\left[\mathrm{~d}(\mathrm{Bw}, \mathrm{Hw})+\mathrm{d}\left(\mathrm{Bw}_{,} \mathrm{Gx}_{2 \mathrm{n}}\right)\right]\right\} \forall \mathrm{n} .
\end{gathered}
$$

Case $1: \mathrm{d}(\mathrm{u}, \mathrm{Bw}) \leq \mathrm{se}_{\mathrm{n}}+\frac{\mathrm{sb}}{2}\left[\mathrm{~d}\left(\mathrm{Gx}_{2 \mathrm{n}}, \mathrm{Hw}\right)+\mathrm{d}\left(\mathrm{Ax}_{2 \mathrm{n}}, \mathrm{Gx}_{2 \mathrm{n}}\right)\right]$

$$
\begin{aligned}
& \leq \mathrm{se}_{\mathrm{n}}+\frac{\mathrm{sb}}{2}\left[\mathrm{~d}\left(\mathrm{Gx}_{2 \mathrm{n}}, \mathrm{u}\right)\right]+\frac{\mathrm{sb}}{2}\left[\operatorname{sd}\left(\mathrm{Ax}_{2 \mathrm{n}}, \mathrm{u}\right)+\mathrm{sd}\left(\mathrm{u}, \mathrm{Gx}_{2 \mathrm{n}}\right)\right] \\
& \leq \mathrm{se}_{\mathrm{n}}+\frac{\mathrm{sb}}{2} \mathrm{e}_{\mathrm{n}+1}+\frac{\mathrm{s}^{2} \mathrm{~b}}{2} \mathrm{e}_{\mathrm{n}}+\frac{\mathrm{s}^{2} \mathrm{~b}}{2} \mathrm{e}_{\mathrm{n}} \\
& \leq \mathrm{s}\left(1+\frac{b}{2}+\mathrm{sb}\right) \mathrm{e}_{\mathrm{n}}
\end{aligned}
$$

Case 2: $d(u, B w) \leq \mathrm{se}_{\mathrm{n}}+\frac{\mathrm{sb}}{2}\left[\mathrm{~d}\left(\mathrm{Gx}_{2 \mathrm{n}}, \mathrm{Hw}\right)+\mathrm{d}(\mathrm{Bw}, \mathrm{Hw})\right]$

$$
\begin{aligned}
\leq \mathrm{se}_{\mathrm{n}}+\frac{\mathrm{sb}}{2}\left[\mathrm{~d}\left(\mathrm{Gx}_{2 \mathrm{n}}, \mathrm{u}\right)\right]+\frac{\mathrm{sb}}{2}[\mathrm{~d}(\mathrm{Bw}, \mathrm{u})] \\
\left(1-\frac{s b}{2}\right) \mathrm{d}(\mathrm{u}, \mathrm{Bw}) \leq \mathrm{se}_{\mathrm{n}}+\frac{\mathrm{sb}}{2} \mathrm{e}_{\mathrm{n}} \\
\mathrm{d}(\mathrm{u}, \mathrm{Bw}) \leq \frac{s\left(\frac{b}{2}+1\right)}{\left(1-\frac{s b}{2}\right)} \mathrm{e}_{\mathrm{n}}
\end{aligned}
$$

Case $3: d(u, B w) \leq \mathrm{se}_{\mathrm{n}}+\frac{\mathrm{sb}}{2}\left[\mathrm{~d}\left(\mathrm{Gx}_{2 \mathrm{n}}, \mathrm{Hw}\right)+\mathrm{d}\left(\mathrm{Ax}_{2 \mathrm{n}}, \mathrm{Hw}\right)\right]$

$$
\begin{aligned}
& \leq \mathrm{se}_{\mathrm{n}}+\frac{\mathrm{sb}}{2}\left[\mathrm{~d}\left(\mathrm{Gx}_{2 \mathrm{n}}, \mathrm{u}\right)\right]+\frac{\mathrm{sb}}{2}\left[\mathrm{~d}\left(\mathrm{Ax}_{2 \mathrm{n}}, \mathrm{u}\right)\right] \\
& \leq \mathrm{se}_{\mathrm{n}}+\frac{\mathrm{sb}}{2} \mathrm{e}_{\mathrm{n}}+\frac{\mathrm{sb}}{2} \mathrm{e}_{\mathrm{n}} \leq \mathrm{s}(1+\mathrm{b}) \mathrm{e}_{\mathrm{n}}
\end{aligned}
$$


Case $4: \mathrm{d}(\mathrm{u}, \mathrm{Bw}) \leq \mathrm{se}_{\mathrm{n}}+\frac{\mathrm{sb}}{2}\left[\mathrm{~d}\left(\mathrm{Gx}_{2 \mathrm{n}}, \mathrm{Hw}\right)+\mathrm{d}\left(\mathrm{Bw}, \mathrm{Gx}_{2 \mathrm{n}}\right)\right]$

$$
\begin{aligned}
\leq \mathrm{se}_{\mathrm{n}}+\frac{\mathrm{sb}}{2}\left[\mathrm{~d}\left(\mathrm{Gx}_{2 \mathrm{n}}, \mathrm{u}\right)\right]+\frac{\mathrm{sb}}{2}\left[\mathrm{sd}(\mathrm{Bw}, \mathrm{u})+\mathrm{sd}\left(\mathrm{u}, \mathrm{Gx}_{2 \mathrm{n}}\right)\right] \\
\left(1-\frac{s^{2} b}{2}\right) \mathrm{d}(\mathrm{u}, \mathrm{Bw}) \leq \mathrm{se}_{\mathrm{n}}+\frac{\mathrm{sb}}{2} \mathrm{e}_{\mathrm{n}}+\frac{\mathrm{s}^{2} \mathrm{~b}}{2} \mathrm{e}_{\mathrm{n}} \\
\mathrm{d}(\mathrm{u}, \mathrm{Bw}) \leq \mathrm{s}\left(\frac{1+\frac{b}{2}+\frac{s b}{2}}{1-\frac{s^{2} b}{2}}\right) \mathrm{e}_{\mathrm{n}}
\end{aligned}
$$

Case $5: d(u, B w) \leq e_{n}+\frac{s b}{2}\left[d(B w, H w)+d\left(A x_{2 n}, H w\right)\right]$

$$
\begin{aligned}
& \leq \mathrm{se}_{\mathrm{n}}+\frac{\mathrm{sb}}{2}[\mathrm{~d}(\mathrm{Bw}, \mathrm{u})]+\frac{\mathrm{sb}}{2}\left[\mathrm{~d}\left(\mathrm{Ax}_{2 \mathrm{n}}, \mathrm{u}\right)\right] \\
\mathrm{d}(\mathrm{u}, \mathrm{Bw}) & \leq \frac{s\left(\frac{b}{2}+1\right)}{\left(1-\frac{s b}{2}\right)} \mathrm{e}_{\mathrm{n}}
\end{aligned}
$$

Case $6: d(u, B w) \leq s e_{n}+\frac{s b}{2}\left[d\left(A x_{2 n}, H w\right)+d\left(B w, G_{2 n}\right)\right]$

$$
\begin{aligned}
\leq \mathrm{se}_{\mathrm{n}}+\frac{\mathrm{sb}}{2}\left[\mathrm{~d}\left(\mathrm{Ax}_{2 \mathrm{n}}, \mathrm{u}\right)\right]+\frac{\mathrm{sb}}{2}\left[\mathrm{sd}(\mathrm{Bw}, \mathrm{u})+\mathrm{sd}\left(\mathrm{u}, \mathrm{Gx}_{2 \mathrm{n}}\right)\right] \\
\left(1-\frac{s^{2} b}{2}\right) \mathrm{d}(\mathrm{u}, \mathrm{Bw}) \leq \mathrm{se}_{\mathrm{n}}+\frac{\mathrm{sb}}{2} \mathrm{e}_{\mathrm{n}}+\frac{\mathrm{s}^{2} \mathrm{~b}}{2} \mathrm{e}_{\mathrm{n}} \\
\mathrm{d}(\mathrm{u}, \mathrm{Bw}) \leq \mathrm{s}\left(\frac{1+\frac{b}{2}+\frac{s b}{2}}{1-\frac{s^{2} b}{2}}\right) \mathrm{e}_{\mathrm{n}}
\end{aligned}
$$

Case $7: d(u, B w) \leq e_{n}+\frac{s b}{2}\left[d(B w, H w)+d\left(B w, G x_{2 n}\right)\right]$

$$
\begin{gathered}
\leq \mathrm{se}_{\mathrm{n}}+\frac{\mathrm{sb}}{2}[\mathrm{~d}(\mathrm{Bw}, \mathrm{u})]+\frac{\mathrm{sb}}{2}\left[\mathrm{sd}(\mathrm{Bw}, \mathrm{u})+\operatorname{sd}\left(\mathrm{u}, \mathrm{Gx}_{2 \mathrm{n}}\right)\right] \\
\left(1-\frac{s b}{2}-\frac{s^{2} b}{2}\right) \mathrm{d}(\mathrm{u}, \mathrm{Bw}) \leq \mathrm{se}_{\mathrm{n}}+\frac{\mathrm{s}^{2} \mathrm{~b}}{2} \mathrm{e}_{\mathrm{n}} \\
\mathrm{d}(\mathrm{u}, \mathrm{Bw}) \leq \frac{\mathrm{s}\left(1+\frac{\mathrm{sb}}{2}\right)}{\left(1-\frac{\mathrm{sb}}{2}-\frac{\mathrm{s}^{2} \mathrm{~b}}{2}\right)} \mathrm{e}_{\mathrm{n}}
\end{gathered}
$$

It is given that g.l.b. of sequences on the right hand side are zero, Then $\mathrm{d}(\mathrm{u}, \mathrm{Bw})=0$, i.e. $\mathrm{Bw}=\mathrm{u}$. Therefore, $\mathrm{Bw}=\mathrm{Hw}=\mathrm{u}$. Thus $\{\mathrm{A}, \mathrm{G}\}$ and $\{\mathrm{B}, \mathrm{H}\}$ have common point of coincidence in $\mathrm{X}$.

It is given that $\{A, G\}$ and $\{B, H\}$ are weakly compatible,

$$
\begin{aligned}
& \mathrm{Au}=\mathrm{AGv}=\mathrm{GAv}=\mathrm{Gu}=\mathrm{m}_{1} \text { (say) and } \\
& \mathrm{Bu}=\mathrm{BHw}=\mathrm{HBw}=\mathrm{Hu}=\mathrm{m}_{2} \text { (say) }
\end{aligned}
$$

Now, $d\left(m_{1}, m_{2}\right)=d(A u, B u) \leq b_{u, u}(A, B, G, H)$ 
where

$$
\begin{gathered}
\mathrm{N}_{\mathrm{u}, \mathrm{u}}(\mathrm{A}, \mathrm{B}, \mathrm{G}, \mathrm{H}) \in\left\{\frac{1}{2}[\mathrm{~d}(\mathrm{Gu}, \mathrm{Hu})+\mathrm{d}(\mathrm{Au}, \mathrm{Gu})], \frac{1}{2}[\mathrm{~d}(\mathrm{Gu}, \mathrm{Hu})+\mathrm{d}(\mathrm{Bu}, \mathrm{Hu})],\right. \\
\frac{1}{2}[\mathrm{~d}(\mathrm{Gu}, \mathrm{Hu})+\mathrm{d}(\mathrm{Au}, \mathrm{Hu})], \frac{1}{2}[\mathrm{~d}(\mathrm{Gu}, \mathrm{Hu})+\mathrm{d}(\mathrm{Bu}, \mathrm{Gu})], \frac{1}{2}[\mathrm{~d}(\mathrm{Bu}, \mathrm{Hu})+\mathrm{d}(\mathrm{Au}, \mathrm{Hu})], \\
\left.\frac{1}{2}[\mathrm{~d}(\mathrm{Au}, \mathrm{Hu})+\mathrm{d}(\mathrm{Bu}, \mathrm{Gu})], \frac{1}{2}[\mathrm{~d}(\mathrm{Bu}, \mathrm{Hu})+\mathrm{d}(\mathrm{Bu}, \mathrm{Gu})]\right\} \forall \mathrm{n} .
\end{gathered}
$$

If $\{\mathrm{A}, \mathrm{G}\}$ and $\{\mathrm{B}, \mathrm{H}\}$ are weakly compatible, then by Lemma1.18, $\mathrm{u}$ is the unique common fixed point of $\mathrm{A}, \mathrm{B}, \mathrm{G}$ and $\mathrm{H}$.

\section{ACKNOWLEDGMENT}

The authors are thankful to the referee for valuable suggestions for the improvement of this paper.

\section{REFERENCES}

[1] M.Abbas, G.Jungck," Common fixed point results for noncommuting mappings without continuity in cone metric spaces", J. Math. Anal. Appl., 341 pp 416-420, 2008

[2] M.Abbas, Rhoades and T.Nazir,",Common fixed point results for four maps in cone metric spaces", Appl. Math. Comput.,216 pp $80-86,2010$

[3] C.D.Aliprantis, K.C.Border, Infinite Dimensional Analysis ,Third Edition, Springer-Verlag, Berlin, 2006

[4] I.Altun, C.Cevik," Some common fixed point theorems in vector metric spaces", Filomat, 25(1), pp 105-113, 2011

[5] I.A.Bakhtin," The contraction mapping principle in quasi-metric spaces", Funct. Anal. Unianowsk Gos. Ped., 30 pp 26-37, 1989

[6] Cevik, I. Altun", Vector metric spaces and some properties", Topol. Methods. Nonlinear Anal., 34(2) pp 375-382, 2009

[7] G. Jungck, B. E. Rhoades, "Fixed point theorems for occasionally weakly compatible mappings", Fixed point theory, 7 (2) pp 287 296, 2006

[8] G. Jungck, "Common fixed point for commuting and compatible maps on compacta,", Proc. Amer. Math. Soc., 103 pp 977-983 , 1988

[9] M.Kamra, S.Kumar and K. Sarita," Some Fixed Point Theorems for Self Mappings on Vector b-metric Spaces", Glob. J. Pure Appl. Math., vol.14, Number 11,pp 1489-1507, 2018

[10] M.Kir, H.Kiziltunc," On some well known fixed point theorems in b-metric spaces", Turkish J. Anal. Number Theory, No.1, Pp 1316,2013

[11] W.A.J.Luxemburg, A.C.Zannen, Riesz Spaces, North-Holland Publishing Company, Amsterdam, 1971.

[12] P.K.Mishra, S. Sachdeva and S.K. Banerjee," Some fixed point theorems in b-metric space”, Turkish J. Anal. Number Theory, Vol. 2, No. 1 pp 19-22, 2014

[13] I.R.Petre, "Fixed point theorems in E-b-metric space"s, J. Nonlinear Sci. Appl., 7 pp 264-271, 2014

[14] G.S.Rad, I.Altun," Common fixed point results on vector metric spaces", J.Linear Topol. Algebra, Vol 5 pp 29-30, 2016

[15] H. Rahimi, M. Abbas and G. S. Rad," Common fixed point results for four mappings on ordered vector metric spaces", Filomat, 29:4 pp 865-878, 2015

[16] H.Rahimi , B.E.Rohades," Fixed point theorems for weakly compatible mappings in cone metric type space", Miskolc Math. Notes, 14(1) pp 233-243, 2013. 